\title{
Continuum model of the twisted graphene bilayer
}

\author{
J. M. B. Lopes dos Santos* \\ CFP and Departamento de Física e Astronomia, Faculdade de Ciências, Universidade do Porto, 4169-007 Porto, Portugal
}

\author{
N. M. R. Peres \\ Graphene Research Center and Department of Physics, National University of Singapore, 2 Science Dr. 3, Singapore 117542 and \\ Centro de Física and Departamento de Física, Universidade do Minho, P-4710-057, Braga, Portugal \\ A. H. Castro Neto \\ Graphene Research Center and Department of Physics, National University of Singapore, 2 Science Dr. 3, Singapore 117542
}

(Received 8 August 2012; published 25 October 2012)

\begin{abstract}
The continuum model of the twisted graphene bilayer [Lopes dos Santos, Peres, and Castro Neto, Phys. Rev. Lett. 99, 256802 (2007)] is extended to include all types of commensurate structures. The essential ingredient of the model, the Fourier components of the spatially modulated hopping amplitudes, can be calculated analytically for any type of commensurate structures in the low-twist-angle limit. We show that the Fourier components that could give rise to a gap in the sublattice exchange symmetric (SE-even) structures discussed by Mele [Phys. Rev. B 81, 161405 (2010)] vanish linearly with angle, whereas the amplitudes that saturate to finite values, as $\theta \rightarrow 0$, ensure that all low-angle structures share essentially the same physics. We extend our previous calculations beyond the validity of perturbation theory to discuss the disappearance of Dirac cone structure at angles below $\theta \lesssim 1^{\circ}$.
\end{abstract}

DOI: 10.1103/PhysRevB.86.155449

PACS number(s): 73.21.Ac, 73.22.Pr

\section{INTRODUCTION}

Barely a year after the discovery of a new form of quantization of the Hall effect in graphene monolayers, ${ }^{1-4}$ the bilayer attracted considerable attention by displaying yet another type of quantum Hall effect. ${ }^{2}$ Experimental and theoretical studies quickly followed on the electronic structure, ${ }^{5}$ Landau-level spectrum, ${ }^{6}$ transport, ${ }^{7-11}$ disorder, and interactions. ${ }^{12,13}$

These early studies focused on the $A B$ stacked bilayer. ${ }^{14}$ Unlike the monolayer, in which carriers near the Fermi level behave like massless fermions, the $A B$ stacked bilayer has quadratic dispersion near the Fermi level (for undoped samples). It is gapless, as the monolayer, but only in the absence of a perpendicular electric field. An important feature of this system is the existence of a variable energy gap induced by an external electric field perpendicular to the layers. ${ }^{15,16}$

The first experimental indications of the existence of rotational disorder in ultrathin graphite films came from films grown on the $4 \mathrm{H}-\mathrm{SiC}(000 \overline{1})$ (carbon side) of $\mathrm{SiC}$ crystals; ${ }^{17}$ however, it had been known for years that in graphite crystals, the top layer is often found rotated with respect to the underlying ones, giving rise to long-wavelength modulations of the scanning tunneling microscopy (STM) signals, displaying as Moiré patterns. ${ }^{18-21}$ Few-layer graphene films grown by chemical vapor deposition methods ${ }^{22-24}$ often show rotations of successive graphene layers. It has also been possible to produce twisted bilayers using mechanically exfoliated samples. ${ }^{25}$

The electronic structure of the twisted bilayer was considered by the authors ${ }^{26}$ in the context of a continuum, Dirac-Weyl equation, description of the two layers, coupled by a spatially modulated hopping. The model predicted the persistence of linear dispersion, with well-defined Dirac cones, as in the monolayer, but with an angle-dependent suppression of the Fermi velocity; it was also predicted that there would be no gap in the presence of a perpendicular electric field. These results were subsequently confirmed experimentally by $\operatorname{Raman}^{25}$ and Landau-level spectroscopy, ${ }^{27}$ and by bandstructure calculations, ${ }^{28,29}$ although the earliest calculations appeared to question the suppression of the Fermi velocity. ${ }^{30,31}$ The most striking confirmation of the electronic structure proposed in Ref. 26 came from the observation, with scanning tunneling spectroscopy, of two low-energy van Hove peaks in the density of states, with a strongly angle-dependent energy difference; these were identified with the occurrence of two saddle points in the band structure. ${ }^{24}$

The continuum description was originally developed for a specific family of commensurate structures, dense in the low-angle limit, in which the relative displacement of corresponding Dirac points in each layer $\Delta \mathbf{K}=\mathbf{K}^{\theta}-\mathbf{K}\left(\mathbf{K}^{\theta}\right.$ is obtained from $\mathbf{K}$ by a rotation of the twist angle between the layers) is not a reciprocal lattice vector of the Moiré superlattice; as a consequence, there is no direct hopping matrix element between these two Dirac points. Mele ${ }^{32}$ considered the commensurability conditions more generally, and pointed out the existence of another family of structures in which $\Delta \mathbf{K}$ is a reciprocal lattice vector of the Moiré superlattice. This matrix element between the Dirac points of the two layers should then give rise to a significant gap, raising the possibility of quite different physics from the one discussed in Ref. 26.

Meanwhile, several authors ${ }^{28,29,33}$ addressed the physics at very low twist angles $\left(\theta \lesssim 1^{\circ}\right)$, finding significant deviations from some of the results presented in our previous work. The continuum model is similar to a quasifree electron calculation, where the kinetic energy scale is $\hbar v_{F} \Delta K=2 \hbar v_{F} K \sin (\theta / 2)$ and the periodic potential scale is given by the interlayer hopping. The original calculation included a minimum set of plane waves, an approximation which is only valid if the kinetic energy scale dominates. 
In this work, we review and extend the continuum model to address these issues. We are able to present a complete analytical calculation of all the Fourier components of the spatially modulated hopping for any family of commensurate structures in the low-angle limit. The structures considered by Mele turn out to be a quasiperiodic repetition of simpler structures of the type we originally considered. The Fourier components of the hopping amplitude that could lead to a gap vanish as the angle decreases, due to an interference effect, whereas other amplitudes saturate, essentially ensuring the low-angle physics of all commensurate structures is the one we discussed previously.

The complete characterization of the Fourier components of the interlayer hopping amplitude allows us to extend the treatment of the continuum model to very small angles. The Fermi velocity vanishes at an angle $\theta \sim 1^{\circ}$ in very good agreement with the results obtained from band-structure calculations; $;^{28,29,33}$ an almost dispersionless band appears at this angle, corresponding to localized states around regions of $A A$ stacking. ${ }^{33}$ Using the continuum model, with only the dominant Fourier amplitude, Bistritzer and MacDonald ${ }^{34}$ showed that at even smaller angles, the Fermi velocity becomes nonzero again, vanishing at a series of "magic angles," of which $\theta \sim 1^{\circ}$ is the first in the series. We present a simple explanation of this observation based on the differences of the band structures of pure $A B$ and pure $A A$ stacked bilayers.

In Sec. II, we review the geometry of commensurate structures in the twisted bilayer in order to establish notation and present an alternative derivation of the results obtained by $\mathrm{Mele}^{32}$ and Shallcross et al. ${ }^{28}$ We formulate the continuum model in Sec. III and present an analytical formulation of the calculation of the Fourier components of the spatially modulated interlayer hopping, valid for small angles and any kind of structure. The main results of the model are presented in Sec. V, followed by a brief summary.

\section{GEOMETRY OF COMMENSURATE STRUCTURES}

The conditions for the commensurability of a Moiré pattern of two rotated honeycomb lattices have already been considered by $\mathrm{Mele}^{32}$ and Shallcross et al. ${ }^{28}$ We review this question, both to establish notation and to present an elementary approach to this question, more directly based on the symmetries of the hexagonal lattice. In this section, we sketch the main argument, leaving details for the Appendix.

The honeycomb (HC) lattice of graphene has an underlying Bravais lattice with basis vectors which we choose as (lattice parameter $a=2.46 \AA$ )

$$
\begin{aligned}
& \mathbf{a}_{1}=a\left\{\frac{1}{2}, \frac{\sqrt{3}}{2}\right\}, \\
& \mathbf{a}_{2}=a\left\{-\frac{1}{2}, \frac{\sqrt{3}}{2}\right\} .
\end{aligned}
$$

This lattice is made up of two sublattices, $A$ and $B$, where $A$ atoms occupy Bravais lattice nodes, and the $B$ are shifted by $\boldsymbol{\delta}_{1}=\left(\mathbf{a}_{1}+\mathbf{a}_{2}\right) / 3$ :

$$
\begin{aligned}
& \mathbf{r}_{A}(m, n)=m \mathbf{a}_{1}+n \mathbf{a}_{2}, \\
& \mathbf{r}_{B}(m, n)=\mathbf{r}_{A}(m, n)+\boldsymbol{\delta}_{1}, \quad m, n \in \mathbb{Z} .
\end{aligned}
$$

In an $A B$ stacked bilayer, there are two such lattices, vertically displaced by $c=3.35 \AA$, with the $B$ atoms of layer $2\left(B_{2}\right)$ with the same horizontal positions as the $A$ atoms in layer $1\left(A_{1}\right)$, $\mathbf{r}_{B 2}(m, n)=\mathbf{r}_{A 1}(m, n)$.

In a twisted bilayer, the layers are rotated relative to each other. We will assume we rotate layer 2 by an angle $\theta$, about a common $A_{1} B_{2}$ horizontal position, that we take to be the origin. A commensurate structure will occur if such a stacking $A_{1} B_{2}$ occurs elsewhere, say at $\mathbf{T}_{1}$; the rotation might as well have been made about that second point, so $\mathbf{T}_{1}$ is a superlattice translation, although not necessarily a primitive vector. For $A_{1} B_{2}$ stacking to occur, a $B_{2}$ site must rotate to an $A_{1}$ site,

$$
k \mathbf{a}_{1}+l \mathbf{a}_{2} \rightarrow m \mathbf{a}_{1}+n \mathbf{a}_{2}, \quad k, l, m, n \in \mathbb{Z}
$$

which can only occur if

$$
k^{2}+l^{2}+k l=m^{2}+n^{2}+m n
$$

since $\left|k \mathbf{a}_{1}+l \mathbf{a}_{2}\right|^{2}=\left(k^{2}+l^{2}+k l\right) a^{2}$.

Shallcross et al. in Ref. 28 present a detailed discussion of the solutions of this Diophantine equation. The same conclusions can be reached by exploring the point symmetries of the hexagonal lattice, namely, the existence of a sixfold rotation axis and of six reflection axes (the lines along the basis vectors $\mathbf{a}_{1}, \mathbf{a}_{2}$ and $\mathbf{a}_{2}-\mathbf{a}_{1}$, and three axis at angles of $\pi / 6$ with these). These symmetries imply that a shell of Bravais lattice sites at a given distance from the origin must be built of groups of two sets of six sites, with position vectors $\mathbf{P}_{i}$ and $\mathbf{Q}_{i}, i=1, \ldots, 6$, such as displayed in Fig. 1(b): the $\mathbf{P}_{i}\left(\mathbf{Q}_{i}\right)$ lie at directions making an angle of $\pi / 3$, and the two sets are related to each other by reflection on the symmetry axis; these two sets may degenerate into one if it occurs on the symmetry axes. Naturally, a rotation of layer 2 by the angle $\theta$ that brings $\mathbf{P}_{1} \rightarrow \mathbf{Q}_{1}$ will leave six $A_{1} B_{2}$ sites at the $\mathbf{Q}$ sites, each defining a lattice translation $\mathbf{T}_{i}$ of a commensurate structure (from origin to $\mathbf{Q}_{i}$ ). The same can be said of the conjugate rotation $\theta^{\prime}=\pi / 3-\theta$ that maps $\mathbf{Q}_{6} \rightarrow \mathbf{P}_{1}$, in which case the lattice translations are defined by the $\mathbf{P}_{i}$. Now, there may be, at a given shell, more than one of these groups of symmetry-related sites. A shell of say 24 atoms will have two such groups $\mathbf{P}_{i}, \mathbf{Q}_{i}$ and $\mathbf{R}_{i}, \mathbf{S}_{i}$. A rotation that, say, maps $\mathbf{R}_{i} \rightarrow \mathbf{Q}_{i}$ must map $\mathbf{S}_{i} \rightarrow \mathbf{P}_{i}$ by symmetry, leaving us with 12 $A_{1} B_{2}$ sites at the same distance from the origin: these lattice

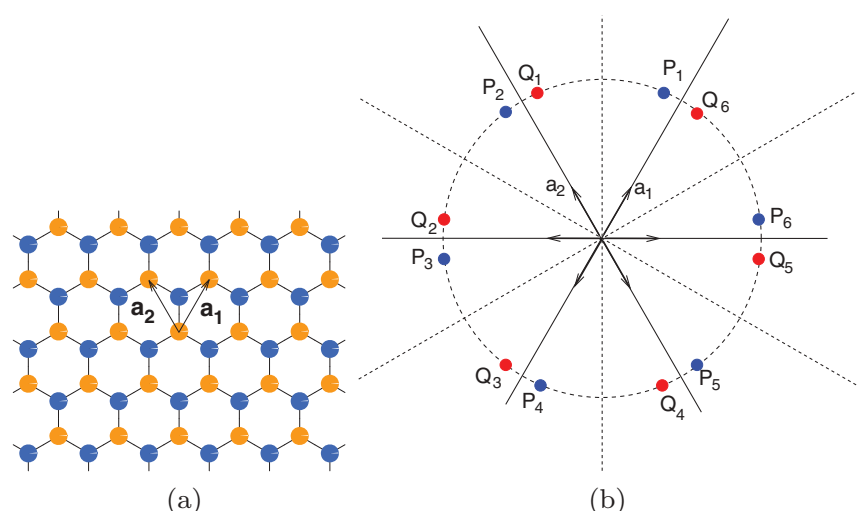

FIG. 1. (Color online) (a) Geometry of the honeycomb lattice. (b) A shell of 12 Bravais lattice sites, their position related by the rotation and reflection symmetries of the hexagonal lattice. 
translations can not be primitive translations since the Bravais superlattice is hexagonal by symmetry, and only has six nearest neighbors. Thus, in order to find all angles of commensuration, and the corresponding primitive vectors, we need only consider rotations that map $\left\{\mathbf{P}_{i}\right\} \rightarrow\left\{\mathbf{Q}_{i}\right\}$ or $\left\{\mathbf{Q}_{i}\right\} \rightarrow\left\{\mathbf{P}_{i}\right\}$, where each of these sets of six points is obtained from the other by reflection about the symmetry axes.

These observations, and some elementary manipulations (see the Appendix) are sufficient to establish the following results for the possible commensurate structures.

Angles: The following equation, with $m$ and $r$ coprime positive integers, defines all possible angles of commensurate structures with $0<\theta<\pi / 3$ :

$$
\cos \theta(m, r)=\frac{3 m^{2}+3 m r+r^{2} / 2}{3 m^{2}+3 m r+r^{2}} .
$$

Primitive vectors: The primitive vectors of the superlattice for a commensurate structure of angle $\theta(m, r)$ are as follows $[\operatorname{gcd}(r, s)$ is the greatest common divisor of $r$ and $s]$ :

(i) If $\operatorname{gcd}(r, 3)=1$,

$$
\left[\begin{array}{l}
\mathbf{t}_{1} \\
\mathbf{t}_{2}
\end{array}\right]=\left[\begin{array}{lc}
m & m+r \\
-(m+r) & 2 m+r
\end{array}\right]\left[\begin{array}{l}
\mathbf{a}_{1} \\
\mathbf{a}_{2}
\end{array}\right] .
$$

(ii) If $\operatorname{gcd}(r, 3)=3$,

$$
\left[\begin{array}{l}
\mathbf{t}_{1} \\
\mathbf{t}_{2}
\end{array}\right]=\left[\begin{array}{lc}
m+\frac{r}{3} & \frac{r}{3} \\
-\frac{r}{3} & m+\frac{2 r}{3}
\end{array}\right]\left[\begin{array}{l}
\mathbf{a}_{1} \\
\mathbf{a}_{2}
\end{array}\right] .
$$

The superlattice unit-cell area is larger than that of graphene by a factor $3 m^{2}+3 m r+r^{2}$ in the first case, and $m^{2}+m r+$ $r^{2} / 3$ in the second one.

These two types of structures can be distinguished both in real and in reciprocal space. ${ }^{32}$ Using the results of the Appendix, it is straightforward to show that in the first case $\operatorname{gcd}(r, 3)=1$, the vertices of the real-space Wigner-Seitz (WS) cell of the superlattice alternate between $B_{1} A_{2}$ sites and hexagon centers; in the second case, each corner of the WS cell is a hexagon center of one layer and an atom of the other. In the reciprocal space, the shift in the Dirac point of the rotated layer $\mathbf{K}^{\theta}-\mathbf{K}$ is a reciprocal lattice vector only in the second case. Mele, ${ }^{32}$ who called attention to these two types of commensurate structures, refers to them as sublattice exchange even (SE-even) when $\operatorname{gcd}(r, 3)=3$ and SE-odd when $\operatorname{gcd}(r, 3)=1$.

\section{CONTINUUM MODEL}

The continuum description of the twisted bilayer was introduced by the authors ${ }^{26}$ in 2007 . A single graphene layer admits an effective description in terms of the Dirac-Weyl equation for states close to one of the Dirac points. ${ }^{3,14} \mathrm{We}$ use this description for the intralayer Hamiltonians in the twisted bilayer, taking into account that layer 2 is rotated with respect to layer 1 by $\theta$. We consider states near the Dirac point $\mathbf{K}=4 \pi(1,0) / 3$ in layer 1 and $\mathbf{K}^{\theta}=(4 \pi / 3)(\cos \theta, \sin \theta)$ in layer 2 . We denote by $\Psi_{i}(r), i=1,2$, the two-component Dirac fields for each of the layers $i=1,2$, and write the momentum as $\mathbf{K}+\mathbf{k}$ in layer 1 and $\mathbf{K}^{\theta}+\mathbf{k}$ in layer 2 .
In momentum space, the intralayer Hamiltonians are 26

$$
\begin{aligned}
& \mathcal{H}_{1}=\hbar \sum_{k} \Psi_{1, \mathbf{k}}^{\dagger} v_{F} \boldsymbol{\tau} \cdot \mathbf{k} \Psi_{1, \mathbf{k}}, \\
& \mathcal{H}_{2}=\hbar \sum_{k} \Psi_{2, \mathbf{k}}^{\dagger} v_{F} \boldsymbol{\tau}^{\theta} \cdot \mathbf{k} \Psi_{2, \mathbf{k}} ;
\end{aligned}
$$

the coordinate axes have been chosen to coincide with those of layer $1, \boldsymbol{\tau}=\left(\tau_{x}, \tau_{y}\right), \boldsymbol{\tau}^{\theta}=e^{+i \theta \tau_{z} / 2} \boldsymbol{\tau} e^{-i \theta \tau_{z} / 2}$, and $\tau_{x}$ and $\tau_{y}$ are Pauli matrices. For the moment, we will ignore coupling between different Dirac valleys $\mathbf{K}, \mathbf{K}^{\theta}$ and $\mathbf{K}^{\prime}=-\mathbf{K}, \mathbf{K}^{\prime \theta}=$ $-\mathbf{K}^{\theta}$; we will return to this point later.

To model the interlayer coupling $\mathcal{H}_{\perp}$, we retain hopping from each site in layer 1 to the closest sites of layer 2 in either sublattice. We denote by $\delta^{\beta^{\prime} \alpha}(\mathbf{r})$ the horizontal (in-plane) displacement from an atom of layer 1 , sublattice $\alpha(\alpha=$ $\left.A_{1}, B_{1}\right)$, and position $\mathbf{r}$ to the closest atom in layer 2 , sublattice $\beta^{\prime}\left(\beta^{\prime}=A_{2}, B_{2}\right)$. The tight-binding interlayer coupling is

$$
\mathcal{H}_{\perp}=\sum_{i, \alpha, \beta^{\prime}} t_{\perp}\left(\delta^{\beta^{\prime} \alpha}\left(\mathbf{r}_{i}\right)\right) c_{\alpha}^{\dagger}\left(\mathbf{r}_{i}\right) c_{\beta^{\prime}}\left(\mathbf{r}_{i}+\delta^{\beta^{\prime} \alpha}\left(\mathbf{r}_{i}\right)\right)+\text { H.c. },
$$

where $t_{\perp}\left(\boldsymbol{\delta}^{\alpha \beta}(\mathbf{r})\right) \equiv t_{\perp}^{\alpha \beta}(\mathbf{r})$ is the interlayer, position-dependent hopping between $p_{z}$ orbitals with a relative displacement $\mathbf{c}_{0}+\boldsymbol{\delta}$, and $c_{\alpha}(\mathbf{r})$ is the destruction operator for the state in sublattice $\alpha$ at horizontal position $\mathbf{r}$.

Denoting by $\Delta \mathbf{K}=\mathbf{K}^{\theta}-\mathbf{K}$ the relative shift between corresponding Dirac wave vectors in the two layers, the usual replacement ${ }^{26} c_{\alpha}(\mathbf{r}) \rightarrow v_{c}^{1 / 2} \psi_{1, \alpha}(\mathbf{r}) \exp (i \mathbf{K} \cdot \mathbf{r})$, where $v_{c}$ is the graphene unit-cell area, leads to

$$
\mathcal{H}_{\perp}=\sum_{\alpha \beta} \int d^{2} r t_{\perp}^{\beta \alpha}(\mathbf{r}) e^{i \mathbf{K}^{\theta} \cdot \delta^{\beta \alpha}(r)} e^{i \Delta \mathbf{K} \cdot \mathbf{r}} \psi_{1, \alpha}^{\dagger}(\mathbf{r}) \psi_{2, \beta}(\mathbf{r})+\text { H.c. }
$$

We used $\psi_{\beta}\left[\mathbf{r}+\delta^{\beta \alpha}(\mathbf{r})\right] \approx \psi_{\alpha}(\mathbf{r})$ since the Dirac fields are slowly varying on the lattice scale.

In Fourier space, it is convenient to define $\phi_{i, \mathbf{k}, \alpha}$ as the Fourier component of $\psi_{i, \alpha}(\mathbf{r})$ for momentum $\mathbf{k} \pm \Delta \mathbf{K} / 2$, the plus sign applying in layer 1 and the minus sign to layer 2 . With this choice, the Dirac fields $\phi_{i, \mathbf{k}, \alpha}$ with the same $\mathbf{k}$ vector in both layers correspond to the same plane waves in the original lattice; the Dirac cones occur at $\mathbf{k}=-\Delta \mathbf{K} / 2$ in layer 1 and $\Delta \mathbf{K} / 2$ in layer 2.

For commensurate structures, the function $t_{\perp}^{\alpha \beta}(\mathbf{r}) \exp$ $\left[i \mathbf{K}^{\theta} \cdot \boldsymbol{\delta}^{\alpha \beta}(\mathbf{r})\right]$ is periodic and has nonzero Fourier components only at the vectors $\mathbf{G}$ of the reciprocal lattice:

$$
\tilde{t}_{\perp}^{\alpha \beta}(\mathbf{G})=\frac{1}{V_{c}} \int_{u c} d^{2} r t_{\perp}^{\alpha \beta}(\mathbf{r}) e^{i \mathbf{K}^{\theta} \cdot \delta^{\alpha \beta}(r)} e^{-i \mathbf{G} \cdot \mathbf{r}} .
$$

The integral is over the unit cell of the superlattice, of area $V_{c}$.

With these definitions, the low-energy effective Hamiltonian, near $\mathbf{K}$, is

$$
\begin{aligned}
\mathcal{H}= & \hbar \sum_{k, \alpha \beta} \phi_{1, \mathbf{k}, \alpha}^{\dagger} v_{F} \boldsymbol{\tau}_{\alpha \beta} \cdot\left(\mathbf{k}+\frac{\Delta \mathbf{K}}{2}\right) \phi_{1, \mathbf{k}, \beta} \\
& +\hbar \sum_{k, \alpha, \beta} \phi_{2, \mathbf{k}, \alpha}^{\dagger} v_{F} \boldsymbol{\tau}_{\alpha \beta}^{\theta} \cdot\left(\mathbf{k}-\frac{\Delta \mathbf{K}}{2}\right) \phi_{2, \mathbf{k}, \beta} \\
& +\left(\sum_{\alpha, \beta} \sum_{\mathbf{k}, \mathbf{G}} \tilde{t}_{\perp}^{\beta \alpha}(\mathbf{G}) \phi_{1, \mathbf{k}+\mathbf{G}, \alpha}^{\dagger} \phi_{2, \mathbf{k}, \beta}+\text { H.c. }\right) .
\end{aligned}
$$


TABLE I. The first and second lines express exact relations between the Fourier amplitudes of the modulated hopping at different reciprocal lattice vectors. In the next two lines, the stated relations have corrections of order $a / L$, where $L$ is the period of the superlattice; $\tilde{t}_{\perp}$ is real.

\begin{tabular}{lccc}
\hline \hline $\mathbf{G}$ & 0 & $-\mathbf{G}_{1}$ & $-\mathbf{G}_{1}-\mathbf{G}_{2}$ \\
\hline$\tilde{t}_{\perp}^{B A}(\mathbf{G})$ & $\tilde{t}_{\perp}$ & $\tilde{t}_{\perp}$ & $\tilde{t}_{\perp}$ \\
$\tilde{t}_{\perp}^{A B}(\mathbf{G})$ & $\tilde{t}_{\perp}$ & $e^{-i 2 \pi / 3} \tilde{t}_{\perp}$ & $e^{i 2 \pi / 3} \tilde{t}_{\perp}$ \\
$\tilde{t}_{\perp}^{A A}(\mathbf{G})$ & $\tilde{t}_{\perp}$ & $e^{i 2 \pi / 3} \tilde{t}_{\perp}$ & $e^{-i 2 \pi / 3} \tilde{t}_{\perp}$ \\
$\tilde{t}_{\perp}^{B B}(\mathbf{G})$ & $\tilde{t}_{\perp}$ & $e^{i 2 \pi / 3} \tilde{t}_{\perp}$ & $e^{-i 2 \pi / 3} \tilde{t}_{\perp}$ \\
\hline \hline
\end{tabular}

Before proceeding, it is perhaps worthwhile to remark that including other interlayer hopping amplitudes does not alter this description in a fundamental way. We would still arrive at a Hamiltonian similar to the one of Eq. (10), but the hopping $t_{\perp}^{\alpha \beta}(\mathbf{r}) \exp \left[i \mathbf{K}^{\theta} \cdot \boldsymbol{\delta}^{\alpha \beta}(\mathbf{r})\right]$ would be replaced by a more complicated expression.

In this formulation, this problem is similar to that of a quasifree electron band problem because each layer has been reduced to a continuum, so that the only periodicity remaining in the problem is that of the Moire superlattice. The most important parameters are then the Fourier amplitudes $\tilde{t}_{\perp}^{\beta \alpha}(\mathbf{G})$ defined by Eq. (12).

The implications of Mele's discussion of SE-even structures $^{32}$ can now be clearly stated. In the SE-odd $r=1$ structures we discussed in $2007, \Delta \mathbf{K}=\left(2 \mathbf{G}_{1}+\mathbf{G}_{2}\right) / 3$ is not a reciprocal lattice vector of the Moiré. There is no matrix element coupling between the Dirac cones $\mathbf{K}$ and $\mathbf{K}^{\theta}$ of the two layers. There is, in fact, a matrix element coupling the different valleys since $\mathbf{K}^{\prime} \theta-\mathbf{K}$ is a reciprocal lattice vector; but, this wave vector has magnitude $\mathcal{O}(1 / a)$, and for Moirés with large periods $L \gg a, t_{\perp}^{\beta \alpha}(\mathbf{r})$ is very slowly varying on the graphene lattice scale, and one would expect such matrix elements to be very small. But, as Mele pointed out, for an SE-even structure $[\operatorname{gcd}(r, 3)=3], \Delta \mathbf{K}=r\left(\mathbf{G}_{1}+\mathbf{G}_{2}\right) / 3$ is a reciprocal lattice vector of magnitude of order $\mathcal{O}(1 / L)$ and there seems to be no a priori reason to neglect it. It lifts the degeneracy between the two Dirac points and leads to a gap. A complete analysis of the Fourier amplitudes, to which we now turn, will allow us to resolve this issue.

\section{CALCULATION OF FOURIER AMPLITUDES}

\section{A. Structures with $r=1$}

We begin by considering the calculation of Fourier amplitudes for $r=1$ structures. Surprisingly, for small angles, the amplitudes for other structures can be reduced to these. In Ref. 26, we stated that in the low-angle limit, and for an $r=1$ structure, the dominant amplitudes are given by the results of Table I. We now give a complete justification of this statement, and show how one can calculate analytically all amplitudes for low angles. We begin by showing how certain symmetries imply relations between the horizontal shifts $\delta^{\beta \alpha}$ for different sublattices.
As stated in Sec. II, three of the six vertices of the WS cell are $B_{1} A_{2}$ sites: for instance,

$$
\mathbf{R}=\frac{2 \mathbf{t}_{1}-\mathbf{t}_{2}}{3}=m \mathbf{a}_{1}+\boldsymbol{\delta}_{1}=(m+1) \mathbf{a}_{1}^{\prime}-\boldsymbol{\delta}_{1}^{\prime} .
$$

Since the origin is an $A_{1} B_{2}$ site, $\mathbf{R}$ is simultaneously a $A_{1} \rightarrow$ $B_{1}$ and a $B_{2} \rightarrow A_{2}$ translation. Therefore, if there is an $A_{1}$ site at $\mathbf{r}$ and $B_{2}$ site at $\mathbf{r}+\delta^{B A}(\mathbf{r})$, there will be a $B_{1}$ site at $\mathbf{r}+\mathbf{R}$ and $A_{2}$ site at $\mathbf{r}+\mathbf{R}+\delta^{B A}(\mathbf{r})$, implying

$$
\mathbf{r}+\boldsymbol{\delta}^{B A}(\mathbf{r})+\mathbf{R}=\mathbf{r}+\mathbf{R}+\boldsymbol{\delta}^{A B}(\mathbf{r}+\mathbf{R}),
$$

and $\boldsymbol{\delta}^{B A}(\mathbf{r})=\boldsymbol{\delta}^{A B}(\mathbf{r}+\mathbf{R})$.

A somewhat more involved symmetry of this structure, namely, invariance under reflection about the origin, sublattice exchange $\left(A_{1} \leftrightarrow B_{1}, A_{2} \leftrightarrow B_{2}\right)$ and translation by $\mathbf{R}=$ $m \mathbf{a}_{1}+\delta_{1}$, leads to a similar relation $\boldsymbol{\delta}^{B B}(\mathbf{r})=-\boldsymbol{\delta}^{A A}(-\mathbf{r}+\mathbf{R})$. These symmetries are exact and imply the following relations for the Fourier amplitudes:

$$
\begin{aligned}
& \tilde{t}_{\perp}^{A B}(\mathbf{G})=e^{-i \mathbf{G} \cdot \mathbf{R}} \tilde{t}_{\perp}^{B A}(\mathbf{G}), \\
& \tilde{t}_{\perp}^{B B}(\mathbf{G})=e^{-i \mathbf{G} \cdot \mathbf{R}}\left[\tilde{t}_{\perp}^{A A}(\mathbf{G})\right]^{*} .
\end{aligned}
$$

With $\mathbf{G}=k \mathbf{G}_{1}+l \mathbf{G}_{2}$, we get $\mathbf{G} \cdot \mathbf{R}=2 \pi(2 k-l) / 3$.

The WS cell also has three vertices which are hexagon centers (see Sec. II); one such vertex is $\left(\mathbf{t}_{1}+\mathbf{t}_{2}\right) / 3$ for $r=1$ structures. This means that $\mathbf{R}=\left(\mathbf{t}_{1}+\mathbf{t}_{2}\right) / 3+\boldsymbol{\delta}_{1}$ is an $A_{1}$ site and $\mathbf{R}^{\prime}=\left(\mathbf{t}_{1}+\mathbf{t}_{2}\right) / 3+\boldsymbol{\delta}_{1}^{\prime}$ is an $A_{2}$ site, and so $\boldsymbol{\delta}_{A A}\left(\mathbf{R}+\boldsymbol{\delta}_{1}\right)=$ $\boldsymbol{\delta}_{1}^{\prime}-\boldsymbol{\delta}_{1} \sim \mathcal{O}(\theta)$. If this were exactly zero, $\mathbf{R}+\boldsymbol{\delta}_{1}$ would be $A_{1} \rightarrow A_{1}$ and $B_{2} \rightarrow A_{2}$ translations, implying

$$
\delta_{A A}(\mathbf{r})=\delta_{B A}\left(\mathbf{r}-\mathbf{R}-\boldsymbol{\delta}_{1}\right)+\mathcal{O}(\theta) .
$$

This leads to

$$
\tilde{t}_{\perp}^{A A}(\mathbf{G}) \approx e^{-i \mathbf{G} \cdot\left(\mathbf{R}+\boldsymbol{\delta}_{1}\right)} \tilde{t}_{\perp}^{B A}(\mathbf{G}) .
$$

As before, $\mathbf{G}=k \mathbf{G}_{1}+l \mathbf{G}_{2}$ and $\mathbf{G} \cdot\left(\mathbf{R}+\boldsymbol{\delta}_{1}\right)=2 \pi(k+$ l) $/ 3+\mathcal{O}(1 / L)$.

These three relations [Eqs. (14) and (15)] express all amplitudes in terms of $\tilde{t}_{\perp}^{B A}(\mathbf{G})$ and have been thoroughly confirmed by numerical evaluation of the Fourier amplitudes by calculating the integrals of Eq. (12) as a lattice sum. For the specific values of $\mathbf{G}$ considered in Table I, they lead to the phase factors relating amplitudes for different sublattices.

Let us now consider the expression for $\tilde{t}_{\perp}^{B A}(\mathbf{G})$, and write it as a lattice sum:

$$
\tilde{t}_{\perp}^{B A}(\mathbf{G})=\frac{1}{N_{c}} \sum_{i \in u c} t_{\perp}\left[\boldsymbol{\delta}^{B A}\left(\mathbf{r}_{i}\right)\right] e^{i \mathbf{K}^{\theta} \cdot \delta_{B A}\left(r_{i}\right)} e^{-i \mathbf{G} \cdot \mathbf{r}_{i}} .
$$

In terms of $\mathbf{G}^{\prime}:=\Delta \mathbf{K}+\mathbf{G}$, we get

$$
\tilde{t}_{\perp}^{B A}(\mathbf{G})=\frac{1}{N_{c}} \sum_{i \in u c} t_{\perp}\left[\boldsymbol{\delta}^{B A}\left(\mathbf{r}_{i}\right)\right] e^{i \mathbf{K}^{\theta} \cdot\left[\mathbf{r}_{i}+\delta_{B A}\left(r_{i}\right)\right]} e^{-i \mathbf{K} \cdot \mathbf{r}_{i}} e^{-i \mathbf{G}^{\prime} \cdot \mathbf{r}_{i}}
$$

In the WS cell of an $r=1$ structure, this simplifies because $\exp \left[i \mathbf{K}^{\theta} \cdot\left(\mathbf{r}+\boldsymbol{\delta}^{B A}\left(\mathbf{r}_{i}\right)\right)\right] \exp [-i \mathbf{K} \cdot \mathbf{r}]=1$ for all sites. It turns out, and this is a property that is exclusive to $r=1$ structures, that the $B_{2}$ site closest to $A_{1}$ at $\mathbf{r}_{A}=m \mathbf{a}_{1}+n \mathbf{a}_{2}$ is at $\mathbf{r}_{B^{\prime}}=$ $m \mathbf{a}_{1}^{\prime}+n \mathbf{a}_{2}^{\prime}$ (same $m$ and $n$ ), so that $\mathbf{K}^{\theta} \cdot \mathbf{r}_{B^{\prime}}=\mathbf{K} \cdot \mathbf{r}_{A}$. As a result,

$$
\tilde{t}_{\perp}^{B A}(\mathbf{G})=\frac{1}{N_{c}} \sum_{i \in u c} t_{\perp}\left[\delta^{B A}\left(\mathbf{r}_{i}\right)\right] e^{-i \mathbf{G}^{\prime} \cdot \mathbf{r}_{i}}
$$


Since $\mathbf{r}_{B_{2}}:=\mathbf{r}_{A_{1}}+\boldsymbol{\delta}^{B A}\left(\mathbf{r}_{A_{1}}\right)=\mathbb{R}(\theta) \cdot \mathbf{r}_{A}$, where $\mathbb{R}(\theta)$ is the rotation matrix, and for small angles $\mathbb{R}(\theta)=\mathbf{1}+d \boldsymbol{\omega} \times \mathbf{r}$, we get $\left|\boldsymbol{\delta}^{B A}(\mathbf{r})\right|=\theta r$. We can therefore approximate the sum of Eq. (18) as an integral:

$$
\begin{aligned}
\tilde{t}_{\perp}^{B A}(\mathbf{G}) & =\frac{1}{N_{c}} \sum_{i \in u c} t_{\perp}\left[\boldsymbol{\delta}^{B A}\left(\mathbf{r}_{i}\right)\right] e^{-i \mathbf{G}^{\prime} \cdot \mathbf{r}_{i}} \\
& \approx \frac{1}{N_{c} \sigma} \int_{u c} d^{2} r t_{\perp}(\theta r) e^{-i G^{\prime} r \cos \phi} .
\end{aligned}
$$

To simplify, we replace the hexagonal unit cell with a circle of the same area $N_{c} \sigma=\sqrt{3} L^{2} / 2$, where $L$ is the superlattice parameter. The radius of the circle is $R_{\mathrm{WS}}=(\sqrt{3} / 2 \pi)^{1 / 2} L$ and

$$
\begin{aligned}
\tilde{t}_{\perp}^{B A}(\mathbf{G}) & =\frac{2}{\sqrt{3} L^{2}} \int_{0}^{R_{\mathrm{Ws}}} d r r t_{\perp}\left(\frac{r}{L}\right) \int_{0}^{2 \pi} d \phi e^{i G^{\prime} r \cos \phi} \\
& =\frac{4 \pi}{\sqrt{3}} \int_{0}^{\left(\frac{\sqrt{3}}{2 \pi}\right)^{1 / 2}} d x x t_{\perp}(x) J_{0}\left(G^{\prime} L x\right),
\end{aligned}
$$

where $J_{0}(x)$ is a Bessel function.

To calculate this integral, we need to parametrize the hopping between $p_{z}$ orbitals as a function of the horizontal shift $\delta$. We express it in the Slater-Koster parameters $V_{p p \sigma}(d)$ and $V_{p p \pi}(d)$, where $d$ is the distance between the two atomic centers $d=\sqrt{c_{0}^{2}+\delta^{2}}$. For the $d$ dependence of $V_{p p \sigma}(d)$ and $V_{p р \pi}(d)$, we used the parametrization of Ref. 35; $V_{p р \pi}\left(a_{0} / \sqrt{3}\right)$ is the in-plane nearest-neighbor hopping $t$, and $V_{p p \sigma}\left(c_{0}\right)$ is the interlayer hopping $t_{\perp}$ in an $A B$ stacked bilayer. The contribution of $V_{р р л}$ turns out to be negligible, and $t_{\perp}(\delta)$ is proportional to $t_{\perp}$ : for $\delta=a_{0} / \sqrt{3}$, the carbon-carbon distance in a layer $t_{\perp}(\delta) / t_{\perp} \approx 0.4$.

With this parametrization, we represent the amplitude as a function of $G^{\prime} L$ in Fig. 2. If $t_{\perp}(\delta)$ were constant, the integral would be proportional to $J_{1}\left(G^{\prime} L\right) /\left(G^{\prime} L\right)$ and decay as $\left(G^{\prime} L\right)^{-3 / 2}$. This is actually the way this amplitude decays, as could be seen by plotting $G^{\prime 3 / 2}$ times the integral. We have calculated numerically, as lattice sums, several amplitudes, using Eq. (16); Fig. 2 shows that the analytical approximation to $\tilde{t}_{\perp}^{B A}(\mathbf{G})$ gives an excellent account of the values found numerically.

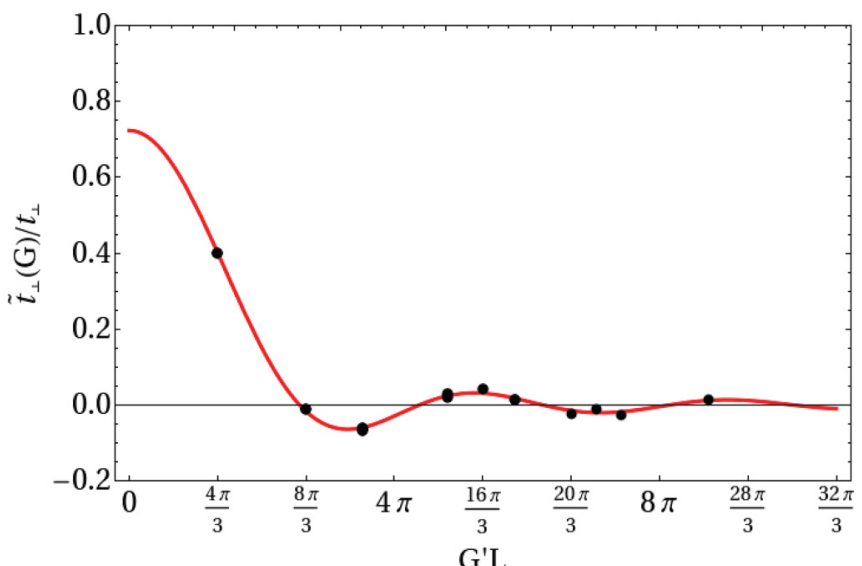

FIG. 2. (Color online) The $\tilde{t}_{\perp}^{B A}(\mathbf{G}) / t_{\perp}$ as a function of $G^{\prime} L$ : the dots are numerically calculated values for a $(m, r)=(10,1)$ structure, with $\theta=3.15^{\circ}$, and the red line is the integral of Eq. (19).
These results are worthy of the following comments:

(i) The three reciprocal lattice vectors selected in Table I, $\mathbf{G}=0, \mathbf{G}=-\mathbf{G}_{1}$, and $\mathbf{G}=-\mathbf{G}_{1}-\mathbf{G}_{2}$, all have $G^{\prime} L=4 \pi / 3$. The corresponding values of $\tilde{t}_{\perp}^{B A}(\mathbf{G})$ are equal, $\tilde{t}_{\perp}^{B A}(\mathbf{G})=$ $0.4 t_{\perp}$; all other reciprocal lattice vectors have larger values of $G^{\prime}$, and the amplitudes are correspondingly smaller; these other amplitudes were ignored in Refs. 26 and 34.

(ii) For a general $\mathbf{G}=k \mathbf{G}_{1}+l \mathbf{G}_{2}, \mathbf{G}^{\prime}=(k+2 / 3) \mathbf{G}_{1}+$ $(l+1 / 3) \mathbf{G}_{2}$. Since $\mathbf{G}_{i} \propto 1 / L, G^{\prime} L$ becomes independent of the angle or rotation. The amplitudes for a given $(k, l)$ become independent of angle for small angles, tending to the values given by our analytical approximation.

(iii) This complete characterization of the Fourier amplitudes allows one, in principle, to include in the calculation of the spectrum as many plane waves as necessary to achieve convergence. The characteristic energy from the in-plane motion is $\hbar v_{F} \Delta K \sim 0.190 \theta$, with the energy in $\mathrm{eV}$ and the angle in degrees, and for small angles one requires more plane waves than those used in Ref. 26. The physics of these small-angle structures has been widely discussed recently in the literature, and we will use these results to discuss it in the framework of the continuum model. But, before that, we consider the calculation of the Fourier amplitudes in other families of commensurate structures.

\section{B. Importance of $r=1$ structures}

In this section, we show that, in the small-angle limit, the $r=1$ structures are special, and determine the physics of all types of commensurate structures. In scanning tunneling microscopy (STM) images, ${ }^{18}$ Moiré patterns appear to satisfy the following relation between period and angle of rotation: $L=a /[2 \sin (\theta / 2)]$. For a general $(m, r)$ structure,

$$
\begin{aligned}
\sin \left(\frac{\theta(m, r)}{2}\right) & =\frac{1}{2} \frac{r}{\sqrt{3 m^{2}+3 m r+r^{2}}}, \\
L(m, r) & =a \sqrt{3 m^{2}+3 m q+q^{2}},
\end{aligned}
$$

where $q=r / \operatorname{gcd}(r, 3)$, so the above relation is only satisfied for $r=1$. The plot $2 L \sin (\theta / 2) / a$ as a function $\theta$, in Fig. 3(a), makes this clear. Remark that all these families of superlattices, with different values of $r$, are dense as $\theta \rightarrow 0$. This means that a very small change in $\theta$, with little effect in the structure in real space, can nevertheless change $L$ by an arbitrary large factor. The implication is that, for very small angles, all commensurate structures are almost periodic repetitions of structures with $r=1$. That is seen very clearly by inspecting visually a few Moiré patterns [see Fig. 3(b)].

Let us show this explicitly for a SE-even structure, $(m, r)$ with $r=3 r^{\prime}$. At one of the corners of the Wigner-Seitz cell,

$$
\mathbf{r}:=\frac{\mathbf{t}_{1}+\mathbf{t}_{2}}{3}=m \boldsymbol{\delta}_{1}+\frac{r}{3} \mathbf{a}_{2}=m \boldsymbol{\delta}_{1}^{\prime}+\frac{r}{3} \mathbf{a}_{1}^{\prime} .
$$

If $m \bmod 3=1$, like in the (7,3) structure in Fig. 3(b), this site has $B_{1}$ atom of layer 1 and a hexagon center of layer 2. Therefore, at $\mathbf{r}-\boldsymbol{\delta}_{1}$ there is an $A_{1}$ site and at $\mathbf{r}-\boldsymbol{\delta}_{1}^{\prime}$, a $B_{2}$ one. This implies that $\boldsymbol{\delta}^{B A}(\mathbf{r})=\boldsymbol{\delta}_{1}^{\prime}-\boldsymbol{\delta}_{1}=\mathcal{O}(\theta)$. If this were zero, $\mathbf{r}$ would be a lattice translation of the Moiré The corresponding structure would be of SE-odd with $m^{\prime}=(m-$ $1) / 3$ and $r^{\prime}=r / 3$. In real space, a SE-even structure $(m, r)$, with $m-1$ divisible by 3 , is then very similar to a SE-odd 


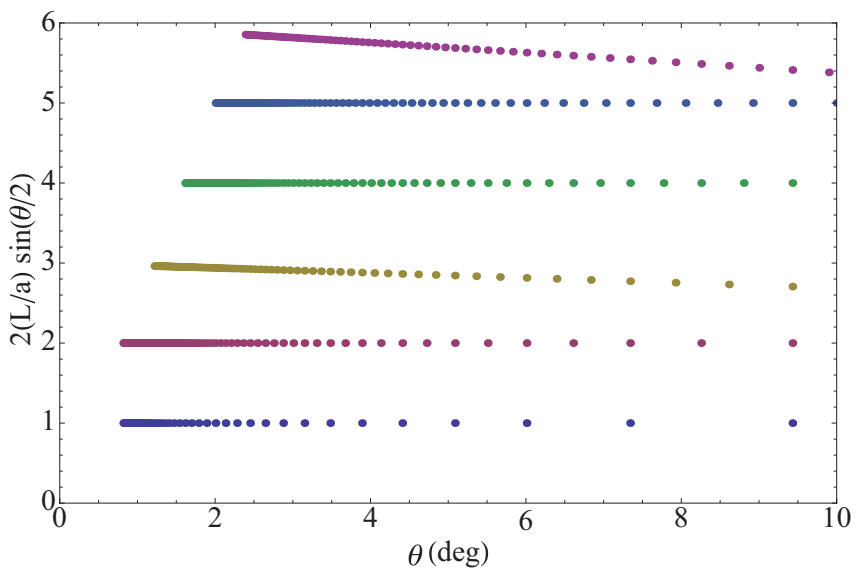

(a)

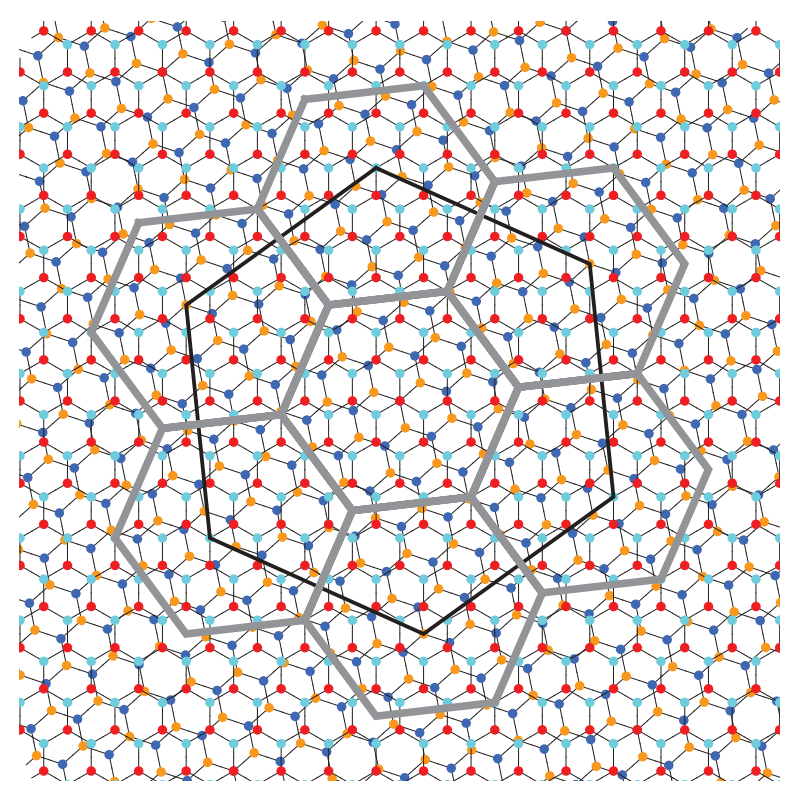

(b)

FIG. 3. (Color online) (a) $2(L / a) \sin (\theta / 2)$ vs $\theta$. The various lines correspond to different values of $r$; the lower line corresponds to the structures with $r=1$. (b) A SE-even structure is almost periodic with the period of a structure with $r=1$; here, a $(m, r)=(7,3)$ is shown overlaid with the Wigner-Seitz cells of $(m, r)=(2,1)$; the black hexagon is the true unit cell of the structure.

with $\left(m^{\prime}, r^{\prime}\right)=[(m-1) / 3, r / 3]$. In the following paragraphs, we refer to these two lattices as $\mathcal{L}$ (SE-even) and $\mathcal{L}$ (SE-odd).

Let us now relate the reciprocal lattice primitive vectors of $\mathcal{L}$ and $\widetilde{\mathcal{L}}$. Using the results of the Appendix, one arrives at

$$
\left[\begin{array}{l}
\widetilde{\mathbf{G}}_{1} \\
\widetilde{\mathbf{G}}_{2}
\end{array}\right]=\left(\left[\begin{array}{ll}
2 & 1 \\
-1 & 1
\end{array}\right]+\mathcal{O}(\theta)\right)\left[\begin{array}{l}
\mathbf{G}_{1} \\
\mathbf{G}_{2}
\end{array}\right] .
$$

Ignore, for the moment, the $\mathcal{O}(\theta)$ corrections. These equations tell us that the real-space basis $\mathcal{L}, \mathbf{t}_{1}, \mathbf{t}_{2}$, are linear combinations with integer coefficients of the basis of $\widetilde{\mathcal{L}}$. In the present case, we have

$$
\left[\begin{array}{l}
\mathbf{t}_{1} \\
\mathbf{t}_{2}
\end{array}\right] \approx\left[\begin{array}{ll}
2 & 1 \\
-1 & 1
\end{array}\right]\left[\begin{array}{l}
\widetilde{\mathbf{t}}_{1} \\
\widetilde{\mathbf{t}}_{2}
\end{array}\right]
$$

In the calculation of $\tilde{t}_{\perp}^{\beta \alpha}(\mathbf{G})$ for the lattice with primitive vectors $\mathbf{t}_{1}, \mathbf{t}_{2}$, we can take into account that the $\delta^{\beta \alpha}\left(\mathbf{r}_{i}\right)$ are (approximately) periodic in $\widetilde{\mathbf{t}}_{1}$ and $\widetilde{\mathbf{t}}_{2}$, and split the sum over $\mathbf{r}_{i}$ in the unit cell $\mathcal{L}$ into a sum over the unit cell of $\widetilde{\mathcal{L}}, \mathbf{r}_{i}^{\prime}$ and a sum over the $n_{c}$ unit cells of $\widetilde{\mathcal{L}}$ contained in the unit cell $\mathcal{L}$ :

$$
\begin{aligned}
\tilde{t}_{\perp}^{\beta \alpha}(\mathbf{G})= & \frac{1}{n_{c}} \sum_{\mathbf{R}_{n}} e^{-i \mathbf{G} \cdot \widetilde{\mathbf{T}}_{n}} \\
& \times \frac{1}{\widetilde{N}_{c}} \sum_{\mathbf{r}_{i}^{\prime} \in \widetilde{u c}} t_{\perp}\left[\delta^{\beta \alpha}\left(\mathbf{r}_{i}^{\prime}+\widetilde{\mathbf{T}}_{n}\right)\right] e^{i \mathbf{K}^{\theta} \cdot \delta^{\beta \alpha}\left(\mathbf{r}_{i}^{\prime}+\mathbf{T}_{n}^{\prime}\right)} e^{-i \mathbf{G} \cdot \mathbf{r}_{i}^{\prime}} \\
\approx & \frac{1}{n_{c}} \sum_{\widetilde{\mathbf{T}}_{n}} e^{-i \mathbf{G} \cdot \widetilde{\mathbf{T}}_{n}} \frac{1}{\widetilde{N}_{c}} \sum_{\mathbf{r}_{i}^{\prime} \in \widetilde{u c}} t_{\perp}\left[\delta^{\beta \alpha}\left(\mathbf{r}_{i}^{\prime}\right)\right] e^{i \mathbf{K}^{\theta} \cdot \delta^{\beta \alpha}\left(\mathbf{r}_{i}^{\prime}\right)} e^{-i \mathbf{G} \cdot \mathbf{r}_{i}^{\prime}} .
\end{aligned}
$$

We achieved a factorization of $\tilde{t}_{\perp}^{\beta \alpha}(\mathbf{G})$ :

$$
\begin{aligned}
\tilde{t}_{\perp}^{\beta \alpha}(\mathbf{G}) & =S(\mathbf{G})\left[\frac{1}{\widetilde{N}_{c}} \sum_{i \in \widetilde{u} c} t_{\perp}\left[\delta^{\beta \alpha}\left(\mathbf{r}_{i}\right)\right] e^{i \mathbf{K}^{\theta} \cdot \delta^{\beta \alpha}\left(\mathbf{r}_{i}\right)} e^{-i \mathbf{G} \cdot \mathbf{r}_{i}}\right], \\
S(\mathbf{G}) & =\frac{1}{n_{c}} \sum_{\widetilde{\mathbf{T}}_{n}} e^{-i \mathbf{G} \cdot \widetilde{\mathbf{T}}_{n}} .
\end{aligned}
$$

The second factor is $\tilde{t}_{\perp}^{\beta \alpha}(\mathbf{G})$ for the lattice $\widetilde{\mathcal{L}}$. As for the structure factor $S(\mathbf{G})$, note that, by definition, $\mathbf{G} \cdot \mathbf{T}=2 m \pi$, if $\mathbf{T}$ is a translation vector of $\mathcal{L}$ (periodic boundary conditions), and, if $\mathbf{G}=\widetilde{\mathbf{G}}$, a reciprocal vector of $\widetilde{\mathcal{L}}, \exp \left[i \mathbf{G} \cdot \widetilde{\mathbf{T}}_{n}\right]=1$. Therefore, we obtain in this approximation

$$
\tilde{t}_{\perp}^{\beta \alpha}(\mathbf{G})=\left[\frac{1}{\widetilde{N}_{c}} \sum_{i \in \widetilde{u c}} t_{\perp}\left[\boldsymbol{\delta}^{\beta \alpha}\left(\mathbf{r}_{i}\right)\right] e^{i \mathbf{K}^{\theta} \cdot \delta^{\beta \alpha}\left(\mathbf{r}_{i}\right)} e^{-i \mathbf{G} \cdot \mathbf{r}_{i}}\right] \delta_{\mathbf{G}, \widetilde{\mathbf{G}}},
$$

where $\widetilde{\mathbf{G}}$ is any reciprocal vector $\widetilde{\mathcal{L}}$. This is a very important result:

(i) it expresses the Fourier amplitudes of SE-even in terms of those of structures with $r=1$, which we calculated in Sec. IV A;

(ii) it states an approximate selection rule, which becomes more accurate as the angle of rotation decreases, allowing us to identify Fourier amplitudes that must tend to zero for small angles.

We have checked this result by numerical calculation of $\tilde{t}_{\perp}^{\beta \alpha}(\mathbf{G})$ for various lattices using Eq. (16). In Fig. 4, each point on the plot has an $x$ coordinate equal to $\tilde{t}_{\perp}^{\beta \alpha}(\widetilde{\mathbf{G}})$, $\widetilde{\mathbf{G}}=k \widetilde{\mathbf{G}}_{1}+l \widetilde{\mathbf{G}}_{2}$, for the $\left(m^{\prime}, r^{\prime}\right)=[(m-1) / 3, r / 3]$ lattice, and a $y$ coordinate $\tilde{t}_{\perp}^{\beta \alpha}(\mathbf{G}), \mathbf{G}=(2 k-l) \mathbf{G}_{1}+(k+l) \mathbf{G}_{2}$ of the SE-even $(m, r)$ lattice; Eq. (26) predicts that these amplitudes should be equal and the agreement is excellent.

The second implication of Eq. (26) concerns the behavior of Fourier amplitudes for which $\mathbf{G}$ is not a reciprocal lattice vector of $\widetilde{\mathcal{L}}$, the $r=1$ superlattice. Of particular interest is $\mathbf{G}=\Delta \mathbf{K}=\mathbf{G}_{1}+\mathbf{G}_{2}$ because it determines the magnitude of the gap in a SE-even structure. In Fig. 5, we show that $\left|\tilde{t}_{\perp}^{\beta \alpha}(\Delta \mathbf{K})\right| \rightarrow 0$ as $\theta \rightarrow 0$, as a result of the vanishing of the structure factor $S(\mathbf{G})$. In other words, there is a destructive interference in the sum of Eq. (16) because of the quasiperiodicity of the hopping amplitudes inside the unit cell of the larger 


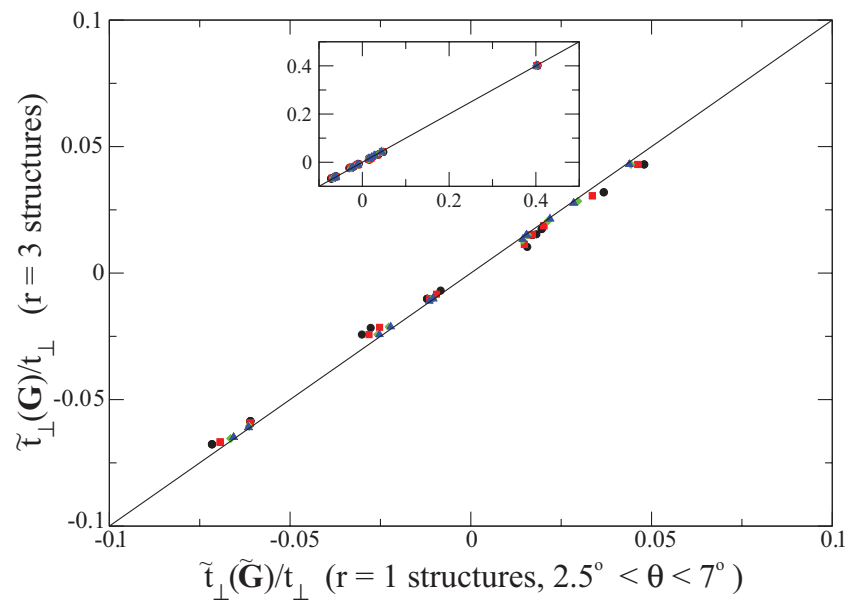

FIG. 4. (Color online) Comparison of Fourier amplitudes of pairs of structures: each point has an $x$ coordinate $\tilde{t}_{\perp}^{\beta \alpha}(\mathbf{G})$ for $\left[(m-1 / 3, r / 3]\right.$ and a $y$ coordinate $\tilde{t}_{\perp}^{\beta \alpha}(\mathbf{G})$ for a SE-even structure $(m, r) . \widetilde{\mathbf{G}}=k \widetilde{\mathbf{G}}_{1}+l \widetilde{\mathbf{G}}_{2}$ and $\mathbf{G}=(2 k-l) \mathbf{G}_{1}+(k+l) \mathbf{G}_{2}$. According to Eq. (26), these amplitudes should be equal. The line is $y=x$, not a fit. The inset has an expanded scale to include the dominant amplitudes $(k, l)=\{(0,0),(-1,0),(-1,-1)\}$. The angles are in the range $2.5^{\circ}<\theta<7.3^{\circ}$.

period lattice. When $\mathbf{G}$ matches a reciprocal lattice vector $\widetilde{\mathbf{G}}$ of the smaller period lattice $S(\mathbf{G}) \approx 1$ (constructive interference), the amplitudes saturate to finite values as $\theta \rightarrow 0$. With this knowledge of the Fourier amplitudes for any structure, we finally address the calculation of the low-energy bands of a small-angle bilayer with a twist.

\section{CONTINUUM MODEL AT LOW ANGLES}

In the absence of the interlayer coupling $\mathcal{H}_{\perp}$, states with energy close to zero occur at $\mathbf{k}=-\Delta \mathbf{K} / 2$ in layer 1 and $\mathbf{k}=$ $+\Delta \mathbf{K} / 2$ in layer 2. The interlayer Hamiltonian $\mathcal{H}_{\perp}$ couples

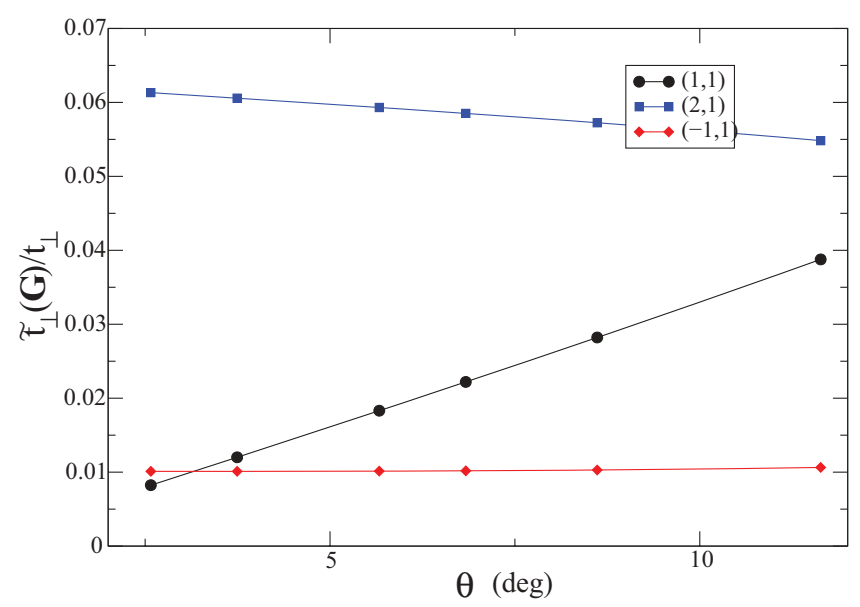

FIG. 5. (Color online) $\left|\tilde{t}_{\perp}^{\beta \alpha}(\mathbf{G})\right| / t_{\perp}$ for $\mathbf{G}=k \mathbf{G}_{1}+l \mathbf{G}_{2}$ for SEeven lattices, with different angles of rotation. For $(k, l)=(-1,1)$ or $(2,1), \mathbf{G}$ is approximately equal to a reciprocal lattice vector of an $r=$ 1 lattice and, $\tilde{t}_{\perp}^{\beta \alpha}(\mathbf{G}) / t_{\perp}$ is almost constant; but for $(k, l)=(1,1)$, which corresponds to $\mathbf{G}=\Delta \mathbf{K}$, the amplitude vanishes linearly with $\theta$. the states of momentum $\mathbf{k}$ in layer 1 to states $\mathbf{k}-\mathbf{G}$, in layer 2 with a matrix element $\tilde{t}_{\perp}^{\beta \alpha}(\mathbf{G})$. The most important Fourier amplitudes (of modulus $\tilde{t}_{\perp}=0.4 t_{\perp}$ ) in $r=1$ structures occur for $\mathbf{G}=0, \mathbf{G}=-\mathbf{G}_{1}$, and $\mathbf{G}=-\mathbf{G}_{1}-\mathbf{G}_{2}$ for which $G^{\prime} L=$ $4 \pi / 3$, where $\mathbf{G}^{\prime}=\mathbf{G}+\Delta \mathbf{K}$ (see Fig. 2). Neglecting other Fourier amplitudes, ${ }^{26}$ the states of momentum $\mathbf{k}$ in layer 1 are coupled directly only to states of layer 2 of momentum $\mathbf{k}, \mathbf{k}+$ $\mathbf{G}_{1}$, and $\mathbf{k}+\mathbf{G}_{1}+\mathbf{G}_{2}$; conversely, the states of momentum $\mathbf{k}$ in layer 2 only couple to states $\mathbf{k}, \mathbf{k}-\mathbf{G}_{1}$, and $\mathbf{k}-\mathbf{G}_{1}-\mathbf{G}_{2}$. To investigate the spectrum at a momentum $\mathbf{k}$ close to zero energy, one can truncate the Hamiltonian to include only these six momentum values (three for each layer) giving a $12 \times 12$ matrix to diagonalize (three momentum values, two layers, and two sub-lattices). ${ }^{26}$ When $\mathbf{k}$ is close to the Dirac cone of one layer, the three momentum values that it couples to lie at the same distance $\Delta K$ from the Dirac point of the opposing layer; we have zero-energy states coupling to two triplets of states at $\pm v_{F} \Delta K$. The spectrum obtained from the diagonalization of the Hamiltonian matrix can be interpreted in a perturbative way when $\tilde{t}_{\perp} / v_{F} \Delta K \ll 1$. This analysis was presented in previous works and will not be repeated here. ${ }^{24,26}$ The main conclusions were (i) the persistence of the Dirac cones, with linear dispersion; (ii) a renormalization of the Fermi velocity, relative to the single layer, which, in perturbation theory, was predicted as $\tilde{v}_{F} / v_{F}=1-9\left[\tilde{t}_{\perp} /\left(\hbar v_{F} \Delta K\right)\right]^{2}\left(v_{F}\right.$ is the singlelayer value); (iii) the appearance of two low-energy van Hove peaks due to the appearance of saddle points in the low-energy bands, arising from the mixing of the two Dirac cones.

In SE-even structures, however, there is a direct matrix element coupling the two Dirac cones; will the physics change relative to SE-odd structures due to the appearance of a gap?

According to Eq. (22), the dominant Fourier amplitudes, in this case, occur for $\mathbf{G}=0, \mathbf{G}=-2 \mathbf{G}_{1}-\mathbf{G}_{2}$, and $\mathbf{G}=-\mathbf{G}_{1}-$ $2 \mathbf{G}_{2}$ since these correspond to $\widetilde{\mathbf{G}}=0,-\widetilde{\mathbf{G}}_{1},-\widetilde{\mathbf{G}}_{1}-\widetilde{\mathbf{G}}_{2}$; on the other hand, $\Delta \mathbf{K}=r\left(\mathbf{G}_{1}+\mathbf{G}_{2}\right) / 3=\mathbf{G}_{1}+\mathbf{G}_{2}$. Therefore, these three dominant amplitudes couple the Dirac point of layer 1 to states of the layer 2 which are shifted from its Dirac point by $-\Delta \mathbf{K}=-\left(\mathbf{G}_{1}+\mathbf{G}_{2}\right),-\Delta \mathbf{K}+2 \mathbf{G}_{1}+\mathbf{G}_{2}=\mathbf{G}_{1}$, and $-\Delta \mathbf{K}+\mathbf{G}_{1}+2 \mathbf{G}_{2}=\mathbf{G}_{2}$; since the angle between $\mathbf{G}_{1}$ and $\mathbf{G}_{2}$ is $2 \pi / 3$, these are three vectors of the same modulus $|\Delta \mathbf{K}|$ at $2 \pi / 3$ angles, and we recognize exactly the same situation as discussed above for the $r=1$ structures: the degeneracy points of each layer couple to two triplets at energies $\pm v_{F} \Delta K$. It is true that, for this structure, there is a direct matrix element coupling the two degeneracy points, corresponding to $\mathbf{G}_{1}+$ $\mathbf{G}_{2}$, which will lift the degeneracy and lead to a gap. However, as we saw in Fig. 5, this matrix element decreases with angle, and below $5^{\circ}$ is under $5 \mathrm{meV}$.

One can say that the differences between the various types of structures in momentum space are somewhat of a red herring. Two structures which are almost identical in real space must display similar physics. The momentum space description can look very different, but the magnitudes of the Fourier amplitudes must ensure similar results.

The perturbation theory in $\tilde{t}_{\perp} /\left(\hbar v_{F} \Delta K\right)$ clearly breaks down for very small angles since, as we have seen, the numerator becomes constant of order $0.4 t_{\perp} \sim 0.1 \mathrm{eV}$ and the denominator is $\hbar v_{F} \Delta K \sim 0.190 \times \theta \mathrm{eV}$ (angle in degrees). This has led some authors ${ }^{28,29,33}$ to question the validity of the 


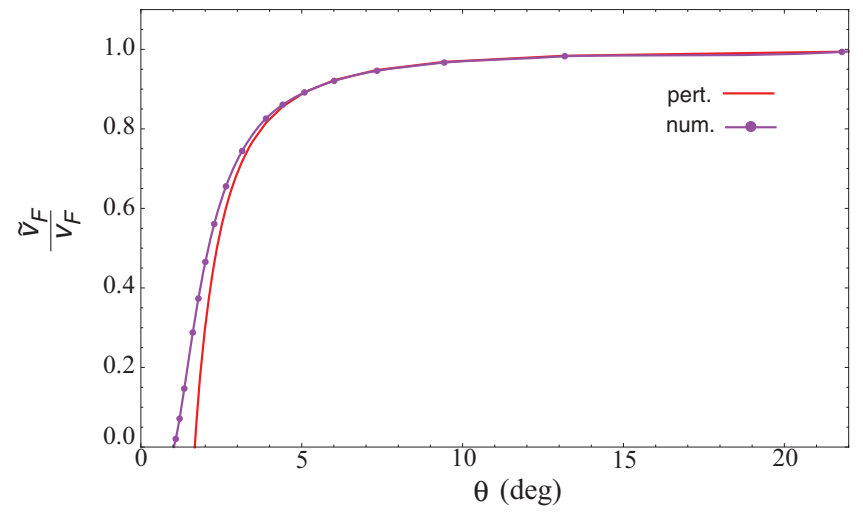

FIG. 6. (Color online) Velocity renormalization, by perturbation theory in $\tilde{t}_{\perp} /\left(\hbar v_{F} \Delta K\right)$, and by the continuum model with numerical diagonalization of the secular equation, with a sufficient set of momentum values for convergence. The latter calculation deviates from the perturbative results as $\left(\hbar v_{F} \Delta K\right)$ becomes comparable to $\tilde{t}_{\perp}$, but is in quite good agreement with band-structure calculations (Refs. 28,29 and 33).

continuum description in the small-angle limit. Band-structure calculations, while confirming the prediction of depressed Fermi velocity $\tilde{v}_{F} / v_{F}=1-9\left[\tilde{t}_{\perp} /\left(\hbar v_{F} \Delta K\right)\right]^{2}$, find deviations from it below about $\theta \approx 5^{\circ}$.

One should not, however, confuse the perturbative result with the continuum model. In fact, the continuum model should be better at smaller angles since the scale of variation of the interlayer hopping becomes larger. What one must do, however, is to include a larger set of plane waves in order to achieve convergence of the low-energy spectrum.

In the following, we present some results for small angles, obtained by diagonalizing numerically the Hamiltonian of Eq. (13), truncated to a finite basis (largest matrix used of $168 \times 168$ ), and including all the required Fourier amplitudes, as given by the analytical expression of Eq. (19). This limit has already been addressed by Bistritzer and MacDonald ${ }^{34}$ in an approximation that includes only the dominant Fourier amplitudes. Some of our calculations, particularly those of the density of states, apparently require larger matrices for convergence than those that these authors claimed to have used.

The results for the ratio of the Fermi velocity to the singlelayer value, as function of $\theta$, are shown in Fig. 6. As expected, for small angles, they deviate from the perturbative result, and compare very well with the values obtained from bandstructure calculations: $:^{28,29,33}$ the Fermi velocity becomes zero at about $\theta \approx 1^{\circ}$.

In Fig. 7(a), we show a density plot of lowest positive energy bands for $\theta=1.79^{\circ}\left(\widetilde{v}_{F} / v_{F} \approx 0.3\right)$; the Dirac cones, as well as the saddle point between them, are clearly visible; at even smaller angles, $\theta=1.20^{\circ}$, the corresponding plot shows an almost flat region in the arc joining the two Dirac cones through the saddle point [Fig. 7(b)]; the range of energies with linear dispersion becomes very small.

The density of states (DOS) is a very convenient tool to check for the presence of Dirac cones in the band structure. If the cones are present, the DOS shows a linear dependence near zero energy, as can be clearly seen in Fig. 8(a) for $\theta=1.79^{\circ}$; for $\theta=1.2^{\circ}$, one can still define a (very small) Fermi velocity, but one should bear in mind that that the range of energies of linear dispersion is contracted to a few $\mathrm{meV}$.

At an even smaller angle, $\theta=1.08^{\circ}$, one observes a sharp peak in the DOS at low energy, corresponding to an almost dispersionless band [Fig. 9(a)], with a barely resolved twopeak structure. Surprisingly, if the angle decreases further, the central band broadens [Fig. 9(b)]. This curious behavior was found by Bistritzer and MacDonald ${ }^{34}$ and characterized as an oscillation of the Fermi velocity. In fact, at the meV resolution of the figure, the DOS is finite between the peaks. It is not clear that a region of linear dispersion even exists, but, if it does, it is so narrow that we prefer to concentrate on this curious variation of the width of the central peak. The diagonalization of the secular equation gives the eigenstates in the momentum basis, as well as the eigenvalues, so it is straightforward to calculate the local density of states. The density plots shown in Fig. 10

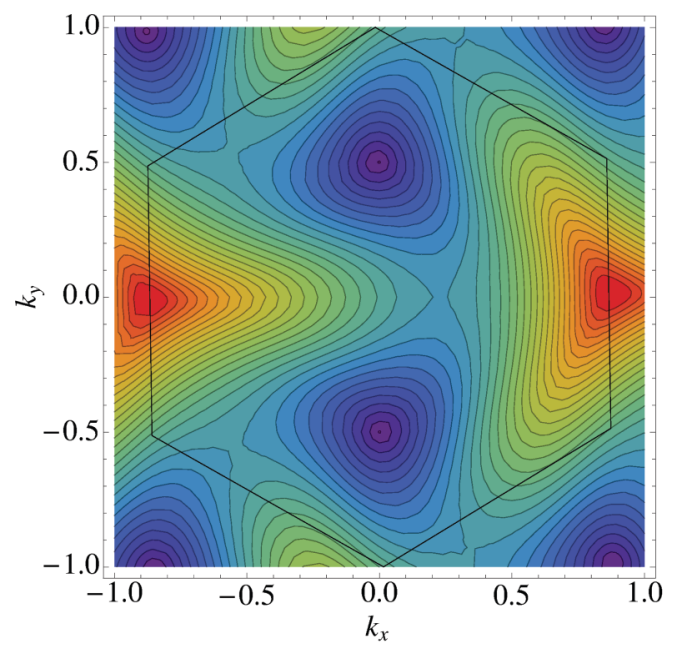

(a)

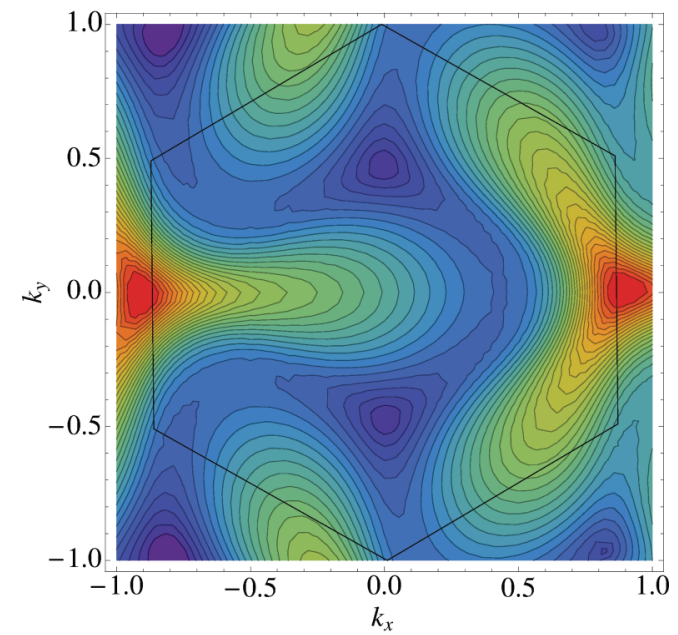

(b)

FIG. 7. (Color online) Contour plot of the first positive energy band; the hexagon is the first Brillouin zone. (a) $\theta=1.79^{\circ}$, the cones are visible, but the saddle point is not located on the line joining the two Dirac cones; (b) $\theta=1.20^{\circ}$, the arc joining the cones through the saddle point has become a very flat valley, and the cones are no longer well defined. 


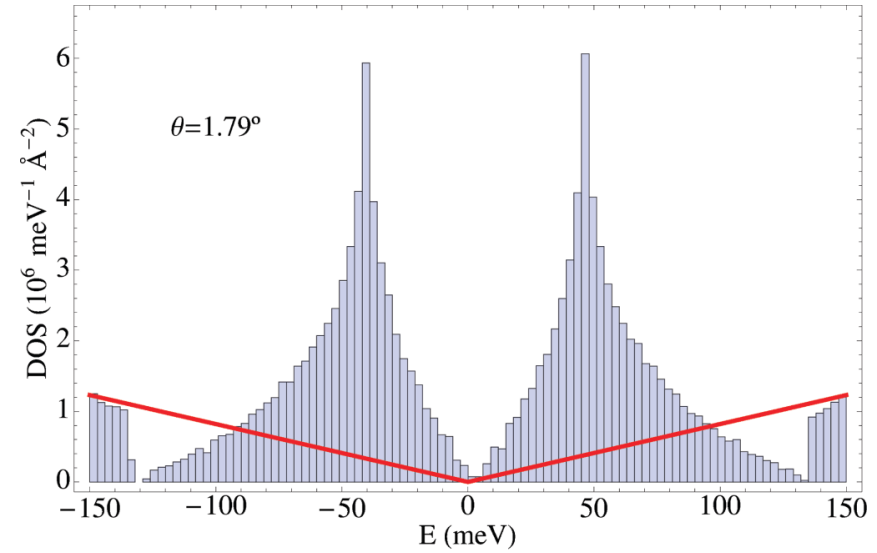

(a)

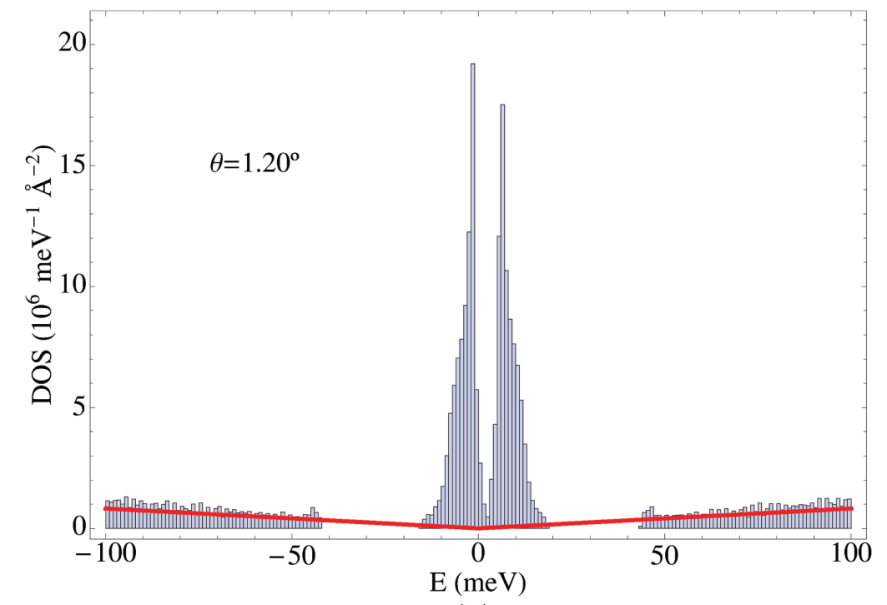

(b)

FIG. 8. (Color online) Densities of states (DOS) for two angles: (a) for $\theta=1.79^{\circ}$, the cones are still well defined and the dispersion shows the usual linear dependence near zero energy; (b) for $\theta=1.2^{\circ}$, there appears to be a finite density of states near zero energy, making if difficult to define a Fermi velocity: the dispersion is no longer linear. The red line is the DOS for two uncoupled layers.

show the local DOS in the superlattice unit cell, integrated over a narrow energy range, close to zero; the dispersionless band is composed of states localized in the $A A$ stacking region, as was found de Laissardière and co-workers. ${ }^{33}$

The reason for this localization, and for the curious fact that the degree of localization can oscillate with angle, can be traced to the difference of band structures of $A A$ and $A B$ (or $B A$ ) bilayers. In a twisted bilayer of small angle, there are well-defined regions of $A A, A B$, and $B A$ stacking, and it is legitimate to reason in terms of the corresponding band structures. The band structure for an $A A$ stacked bilayer, near each Dirac point, is composed of two cones shifted in energy by $\pm t_{\perp}$, corresponding to the bonding and antibonding combinations of $p_{z}$ orbitals in each plane (Fig. 11). As a result, the Fermi surfaces for electrons and holes, at zero energy, are circular with radius $k_{F}=t_{\perp} / \hbar v_{F}$. But, on an $A B$ or $B A$ stacked bilayer at zero energy, the Fermi surface is a point. From conservation of momentum parallel to an $A A \leftrightarrow A B$ interface, one can immediately conclude that there can be no transmission $A A \rightarrow A B(B A)$ for any nonzero angle; in fact, a calculation shows that the transmission coefficient is also

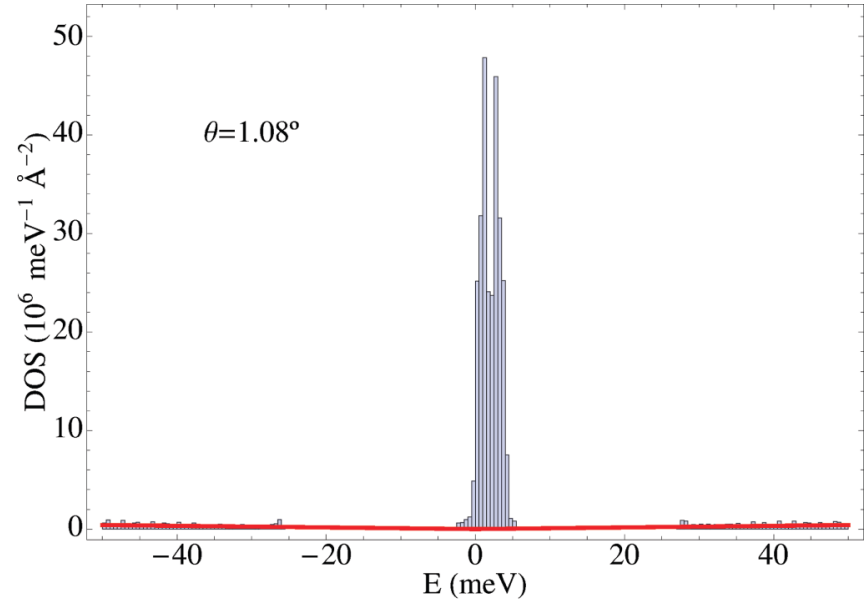

(a)

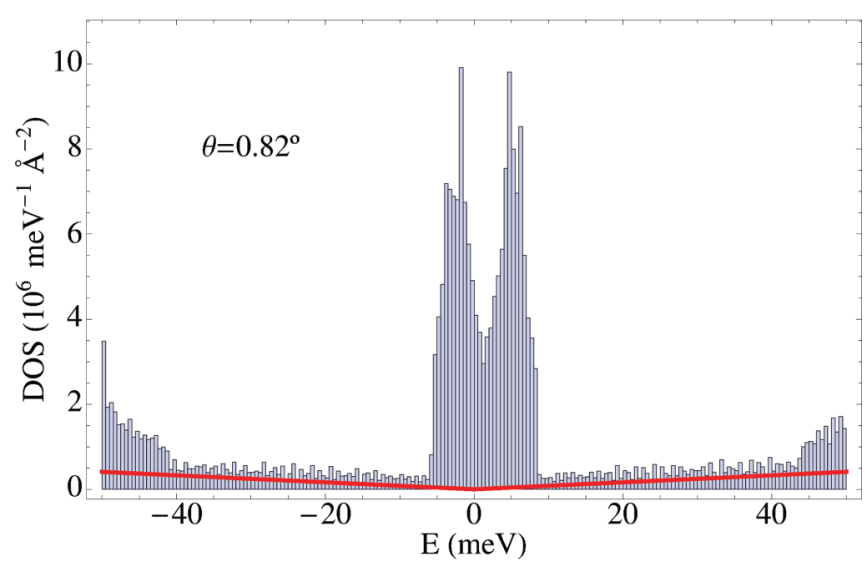

(b)

FIG. 9. (Color online) Densities of states (DOS) for two small angles: (a) for $\theta=1.08^{\circ}$, there is a very sharp central peak, with a barely resolved two-peak structure, corresponding to a flat band of states localized in $A A$ stacking regions of the unit cell; (b) for $\theta=0.87^{\circ}$, the central band is broader, and still displays the twopeak structure, although, at the meV resolution, there is a finite DOS between the peaks, precluding the unambiguous definition of a Fermi velocity.

zero for zero angle of incidence and energy. Since the $A A$ region is enclosed by a hexagon of $A B$ and $B A$ stacking, this raises the possibility of localization of zero-energy states in the $A A$ region. However, this localization only occurs for zero energy; for finite energy, some transmission is possible. Now, a confined $A A$ region will have a discrete spectrum. The energy levels move toward the corresponding Dirac energies $\left( \pm t_{\perp}\right)$ if the size of the unit cell size increases. It is clear then that when a discrete level in an AA region occurs at zero energy, we can expect strong localization and a dispersionless band. If we further decrease the angle, by increasing the unit cell, the discrete level moves away from zero energy, it starts tunneling into the neighboring $A B$ and $B A$ regions, and the low-energy band broadens. This, we believe, is the rather simple explanation for the oscillation of the bandwidth of the central peak in the DOS. De Laissardière and co-workers ${ }^{36}$ recently found confirmation of this explanation in large-scale tight-binding calculations. Another explanation for this same 


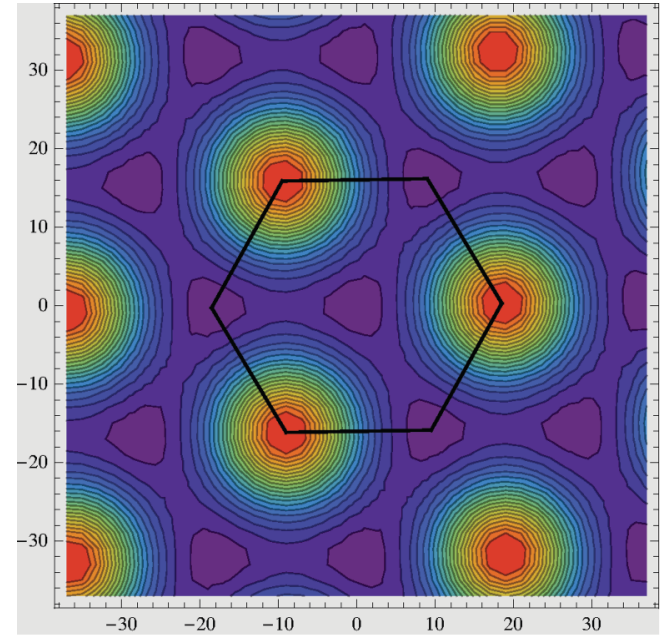

(a)

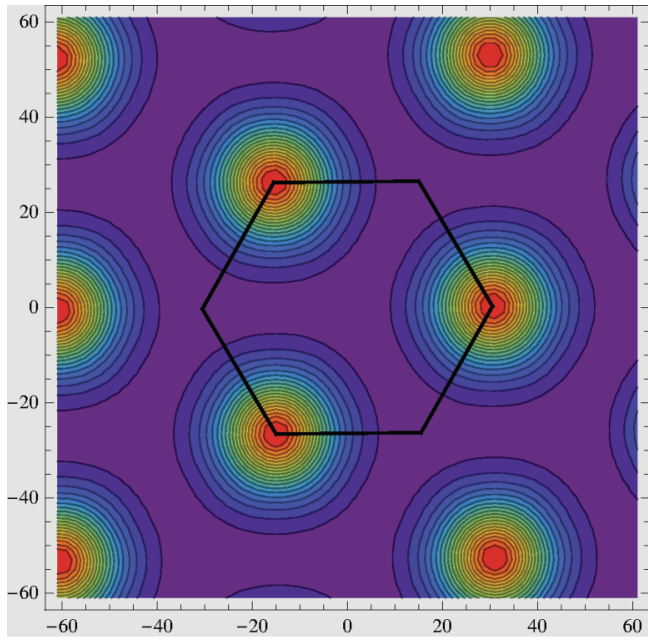

(b)

FIG. 10. (Color online) Density plots of the local density of states, in the Wigner-Seitz cell, integrated for $|\epsilon|<20 \mathrm{meV}$ : (a) $\theta=1.78^{\circ}$, (b) $\theta=1.08^{\circ}$.

oscillation, formulated in terms of non-Abelian effective gauge fields, has recently been proposed. ${ }^{37}$

The extremely flat bands, which Bistritzer and MacDonald $^{34}$ associate with the zeros of the Fermi velocity, correspond to the passage of a confined state in an $A A$ region through energies $\pm t_{\perp}$ above (below) the corresponding Dirac energies (i.e., through zero energy). It has been pointed out by de Laissardière et $a l .{ }^{36}$ that this interpretation is consistent with the values of the magic angles at which flat bands occur. They are roughly described by the formula $\theta_{n}\left({ }^{\circ}\right) \sim 1.05 / n$, $n=1,2, \ldots$ (see Ref. 34); zero-energy states in the $A A$ region have a momentum $k=t_{\perp} / \hbar v_{F}$, so one would expect a quantization condition of the type $k L(\theta) \sim 2 \pi n$, where $L(\theta)$ is the Moiré period, $L(\theta) \approx a / \theta$ for small angles, or

$$
\theta \sim \frac{1}{2 \pi} \frac{t_{\perp}}{\hbar v_{F}} \frac{a}{n}=1.08^{\circ} \times \frac{1}{n}
$$

$\left(t_{\perp} \approx 0.3 \mathrm{eV}\right)$. The double-peak structure originates in the fact that zero energy is equally distant from the electron and hole

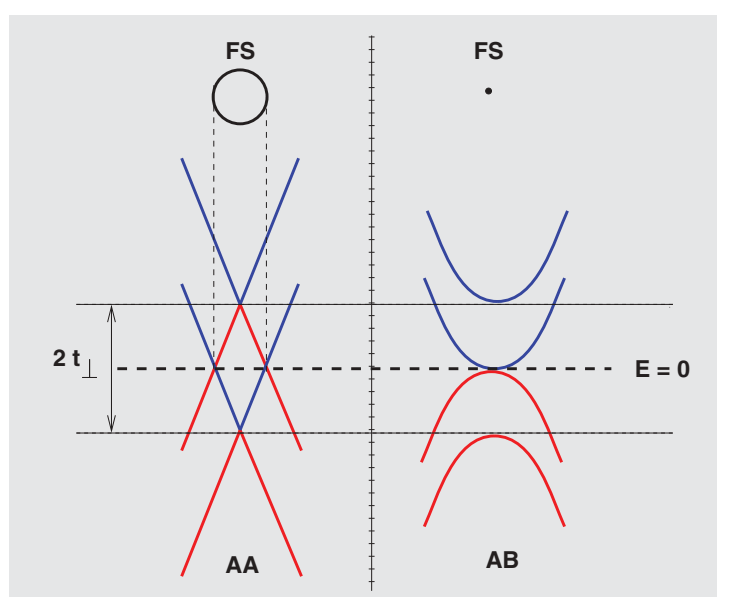

FIG. 11. (Color online) Band structures of pure $A A$ and pure $A B$ stacking bilayers.
Dirac point energies $\left(\mp t_{\perp}\right)$, so states cross zero energy in pairs (one electron and one holelike state) in the $A A$ regions.

\section{DISCUSSION AND CONCLUSIONS}

We have analyzed in detail the continuum description of the twisted bilayer focusing on small-angle structures. We generalized our previous treatment to include all types of commensurate structures, and addressed in particular the possibility of a gapped electronic spectrum for SE-even structures raised by Mele. ${ }^{32}$ We have shown, that for small angles, all commensurate structures are either of the type $r=1$, in which the relation between the period and angle or rotation is that found in STM studies of Moire patterns $L=a /[2 \sin (\theta / 2)]$, or almost periodic repetitions of such structures. As a consequence, even though the momentum space description can be quite different, small-angle commensurate structures share the same physics.

We have achieved a complete analytical characterization of the Fourier components of the spatially modulated hopping amplitudes, which allows a detailed study of very-small-angle structures. This continuum description accounts very well for the renormalization of the Fermi velocity relative to the singlelayer value. The density of states is a revealing tool; if the angle is not too small, two well-defined van Hove peaks appear at low energies, and, near zero energy, the DOS rises linearly, as expected for linear dispersion [Fig. 8(a)]; at $\theta=1.08^{\circ}$, the van Hove peaks are no longer resolved, as the range of linear dispersion shrinks to zero; a low-energy, almost flat, band appears, separated by gaps from the rest of the spectrum (at positive and negative energies). This flat band is formed from states localized in $A A$ stacking regions which, at zero energy, can not tunnel into $A B$ and $B A$ regions. However, if the angle is further decreased, the energy of these localized states changes, and they can start tunneling into the neighboring regions. This explains the oscillation with angle of the bandwidth of the central peak of the density of states. 


\section{ACKNOWLEDGMENTS}

J.M.B.L.S. was supported by Fundação para a Ciência e a Tecnologia (FCT) and is thankful for the hospitality of Boston University and of National University of Singapore. N.M.R.P. was supported by Fundos FEDER through the Programa Operacional Factores de Competitividade-COMPETE and by FCT under Project No. PEst-C/FIS/UI0607/2011, and is thankful for the hospitality of Boston University. A.H.C.N. acknowledges DOE Grant No. DE-FG02-08ER46512, ONR Grant No. MURI N00014-09-1-1063, and the NRF-CRP award "Novel 2D materials with tailored properties: beyond graphene" (R-144-000-295-281).

\section{APPENDIX: GEOMETRY}

Given an arbitrary site of the hexagonal Bravais lattice $\mathbf{P}_{1}=$ $k \mathbf{a}_{1}+l \mathbf{a}_{2}$, the rotational/reflection symmetry implies that it is part of a set of $12\left\{\mathbf{P}_{i}, \mathbf{Q}_{i}: i=1, \ldots, 6\right\}, \pi / 3$ being the angle between directions of consecutive points in $\left\{\mathbf{P}_{i}\right\}$ or in $\left\{\mathbf{Q}_{i}\right\}$, and each of these sets being the image of the other under reflection about the symmetry axes [see Fig. 1(b)]. These two sets merge into one if and only if either $k$ or $l$ is zero or $k=l$. In the main text, we argued that we need only consider rotations that map one of these sets onto its image by reflection, in order to obtain all angles and primitive vectors of commensurate structures. Without loss of generality, we can choose

$$
\begin{aligned}
\mathbf{P}_{1} & =n \mathbf{a}_{1}+m \mathbf{a}_{2}, \\
\mathbf{Q}_{1} & =m \mathbf{a}_{1}+n \mathbf{a}_{2}, \\
\mathbf{Q}_{6} & =(m+n) \mathbf{a}_{1}-m \mathbf{a}_{2}
\end{aligned}
$$

with $n>m>0$; values of $m$ or $n$ zero, or $m=n$, correspond to $\pi / 3$ rotations, that transform an $A B$ stacked bilayer into an $A A$ one [see also Fig. 1(b)]. So, anticlockwise commensurate rotations with angles $0<\theta<\pi / 3$ are of two types:

$$
\begin{aligned}
\theta: & \mathbf{P}_{1} \rightarrow \mathbf{Q}_{1} ; \quad(n, m) \rightarrow(m, n) ; \\
\theta^{\prime}: & \mathbf{Q}_{6}^{\prime} \rightarrow \mathbf{P}_{1}^{\prime} ; \quad(p+q,-p) \rightarrow(q, p) .
\end{aligned}
$$

In the first case, $\mathbf{T}_{1}:=m \mathbf{a}_{1}+n \mathbf{a}_{2}$ is a superlattice translation; in the second, it is $\mathbf{T}^{\prime}=q \mathbf{a}_{1}+p \mathbf{a}_{2}$. We will soon see under what conditions these are primitive vectors. These two rotations are conjugate, $\theta+\theta^{\prime}=\pi / 3$, if $m=p$ and $n=q$.

In the following, it will be useful to to define these rotations in terms of the pair of integers $m, r$ with $r=n-m$, and $p, s$ with $s=q-p$ :

$$
\begin{aligned}
(m+r, m) & \rightarrow(m, m+r), \\
(2 p+s,-p) & \rightarrow(p+s, p) .
\end{aligned}
$$

One easily derives the following results for the angles by taking the scalar product of final and initial vectors

$$
\begin{aligned}
\cos \theta & =\frac{3 m^{2}+3 m r+r^{2} / 2}{3 m^{2}+3 m r+r^{2}} \\
& =\frac{3(m+r / 2)^{2}-(r / 2)^{2}}{3(m+r / 2)^{2}+(r / 2)^{2}}, \\
\cos \theta^{\prime} & =\frac{3 p^{2} / 2+3 p s+s^{2}}{3 p^{2}+3 p s+s^{2}}
\end{aligned}
$$

$$
=\frac{3(3 p / 2+s)^{2}-(3 p / 2)^{2}}{3(3 p / 2+s)^{2}+(3 p / 2)^{2}} .
$$

The second form of each expression makes it clear that the two families define the same set of angles: $\theta=\theta^{\prime}$, if $m / r=$ $s / 3 p$; all angles of commensurate structures are generated [Eq. (A4a)] with $m$ and $r$ positive integers: $\theta^{\prime}(p, s)=\theta(m, r)$ if $m=s$ and $r=3 p$.

Given two positive integers $m, r$, and the angle $\theta(m, r)$ defined by Eq. (A4a), there is a unique set of integers $p, q$ for which one of the following representations

$$
\begin{aligned}
& \cos \theta(m, r)=\frac{3 p^{2}+3 p q+q^{2} / 2}{3 p^{2}+3 p q+q^{2}}, \\
& \cos \theta(m, r)=\frac{3 p^{2} / 2+3 p q+q^{2}}{3 p^{2}+3 p q+q^{2}}
\end{aligned}
$$

has the smallest denominator. If the smallest denominator occurs for the first form, we conclude that $\mathbf{t}_{1}:=p \mathbf{a}_{1}+(p+$ $q) \mathbf{a}_{2}$ is a lattice translation, with the smallest norm (the denominator is $\left|\mathbf{t}_{1}\right|^{2}$ ) and, therefore, a primitive vector. The other can be obtained by a $\pi / 3$ rotation of $\mathbf{t}_{1}$. On the other hand, if the second form has the smallest denominator, then, by the same reasoning, $\mathbf{t}_{1}=(p+q) \mathbf{a}_{1}+p \mathbf{a}_{2}$ is a primitive vector of the super-lattice.

From this point on, we assume that $m, r$ are coprime because otherwise we can always reduce the denominator by factoring out the divisors of $m$ and $r$. If

$$
\frac{3 m^{2}+3 m r+r^{2} / 2}{3 m^{2}+3 m r+r^{2}}=\frac{3 p^{2}+3 p q+q^{2} / 2}{3 p^{2}+3 p q+q^{2}}
$$

and $3 p^{2}+3 p q+q^{2}<3 m^{2}+3 m r+r^{2}$, we must have

$$
\begin{aligned}
3 m^{2}+3 m r+r^{2} / 2 & =\lambda\left(3 p^{2}+3 p q+q^{2} / 2\right), \\
3 m^{2}+3 m r+r^{2} & =\lambda\left(3 p^{2}+3 p q+q^{2}\right),
\end{aligned}
$$

where $\lambda$ is a positive integer. Subtracting these equations, one gets $r^{2}=\lambda q^{2}$, so that $\lambda=s^{2}$, where $s$ is a divisor of $r$. Solving the second equation for $m / s$ gives, recalling that $m, r, p, q$ are positive integers,

$$
\frac{m}{s}=-\frac{q}{2} \pm \frac{1}{2} \sqrt{q^{2}+4 p(p+q)}=p .
$$

So, $s$ must a common divisor of $m$ and $r$, and, since $m, r$ are coprime, $s=1$, and the initial form already has the smallest denominator. An entirely similar argument can applied to reducing to the second form [Eq. (A5b)]. A form with smaller denominator is possible if $r$ is a multiple of 3 , and $(p, q)=(m, r / 3)$.

In conclusion, we can state that if $(m, r)$ are coprime and

$$
\cos \theta=\frac{3 m^{2}+3 m r+r^{2} / 2}{3 m^{2}+3 m r+r^{2}},
$$

the superlattice basis vectors are given by $\mathbf{t}_{i}=\sum_{j} S_{i j} \mathbf{a}_{j}$, and the matrix $\mathbf{S}$ is defined in Eqs. (6) and (7). 
Shallcross et al. define the angles and primitive vectors in terms of two coprime integers $p$ and $q$; their results coincide with these with the following correspondence: if $r$ is odd, $p=r$ and $q=2 m+r$; if $r$ is even, $p=r / 2$ and $q=m+r / 2$.

From these results, one can obtain other useful relations. Since $\mathbf{t}_{1}$ and $\mathbf{t}_{2}$ are lattice translations of both layers, they have equally simple expressions in terms of the primitive vectors of the rotated layers $\mathbf{a}_{1}^{\prime}$ and $\mathbf{a}_{2}^{\prime}$. The transformation between this nonorthogonal basis is

$$
\left[\begin{array}{l}
\mathbf{a}_{1} \\
\mathbf{a}_{2}
\end{array}\right]=\left[\begin{array}{lc}
\cos \theta+\sin \theta / \sqrt{3} & -2 \sin \theta / \sqrt{3} \\
2 \sin \theta / \sqrt{3} & \cos \theta-\sin \theta / \sqrt{3}
\end{array}\right]\left[\begin{array}{l}
\mathbf{a}_{1}^{\prime} \\
\mathbf{a}_{2}^{\prime}
\end{array}\right] .
$$

The rotation matrix can be expressed in terms of $m$ and $r$ using Eq. (A4a), leading to

$$
\left[\begin{array}{l}
\mathbf{t}_{1} \\
\mathbf{t}_{2}
\end{array}\right]=\left[\begin{array}{cc}
m+r & m \\
-m & 2 m+r
\end{array}\right]\left[\begin{array}{l}
\mathbf{a}_{1}^{\prime} \\
\mathbf{a}_{2}^{\prime}
\end{array}\right]
$$

for $\operatorname{gcd}(r, 3)=1$ and

$$
\left[\begin{array}{l}
\mathbf{t}_{1} \\
\mathbf{t}_{2}
\end{array}\right]=\left[\begin{array}{lc}
m+2 r / 3 & -r / 3 \\
r / 3 & m+r / 3
\end{array}\right]\left[\begin{array}{l}
\mathbf{a}_{1}^{\prime} \\
\mathbf{a}_{2}^{\prime}
\end{array}\right]
$$

for $\operatorname{gcd}(r, 3)=3$.

The dual basis of $\left\{\mathbf{a}_{1}, \mathbf{a}_{2}\right\}$ (reciprocal lattice primitive vectors) can be chosen as

$$
\left[\begin{array}{l}
\mathbf{g}_{1} \\
\mathbf{g}_{2}
\end{array}\right]=\frac{4 \pi}{3\left|\mathbf{a}_{1}\right|}\left[\begin{array}{cc}
2 & -1 \\
-1 & 2
\end{array}\right]\left[\begin{array}{l}
\mathbf{a}_{1} \\
\mathbf{a}_{2}
\end{array}\right],
$$

with a similar relation for $\left\{\mathbf{t}_{1}, \mathbf{t}_{2}\right\}$ and its dual basis $\left\{\mathbf{G}_{1}, \mathbf{G}_{2}\right\}$. Knowing that the Dirac points are given as $\mathbf{K}=$ $(4 \pi / 3)\left(\mathbf{a}_{1}-\mathbf{a}_{2}\right)$, and $\mathbf{K}^{\theta}=(4 \pi / 3)\left(\mathbf{a}_{1}^{\prime}-\mathbf{a}_{2}^{\prime}\right)$, one can show, using Eqs. (A9)-(A12), (6), and (7), after some tedious but trivial algebra, the following relations:

$$
\Delta \mathbf{K}^{\theta}:=\mathbf{K}^{\theta}-\mathbf{K}= \begin{cases}\frac{r}{3}\left(2 \mathbf{G}_{1}+\mathbf{G}_{2}\right) & \text { if } \operatorname{gcd}(r, 3)=1, \\ \frac{r}{3}\left(\mathbf{G}_{1}+\mathbf{G}_{2}\right) & \text { if } \operatorname{gcd}(r, 3)=3 .\end{cases}
$$

Note that, in the second case only, $\Delta \mathbf{K}$ is a reciprocal lattice vector.
*Corresponding author: jlsantos@fc.up.pt

${ }^{\dagger}$ On leave from Department of Physics, Boston University, 590 Commonwealth Avenue, Boston, MA 02215.

${ }^{1}$ V. P. Gusynin and S. G. Sharapov, Phys. Rev. Lett. 95, 146801 (2005).

${ }^{2}$ K. S. Novoselov et al., Nat. Phys. 2, 177 (2006).

${ }^{3}$ N. M. R. Peres, F. Guinea, and A. H. Castro Neto, Phys. Rev. B 73, 125411 (2006).

${ }^{4}$ Y. B. Zhang et al., Nature (London) 438, 201 (2005).

${ }^{5}$ T. Ohta, A. Bostwick, T. Seyller, K. Horn, and E. Rotenberg, Science 313, 951 (2006).

${ }^{6}$ E. McCann and V. I. Fal'ko, Phys. Rev. Lett. 96, 086805 (2006).

${ }^{7}$ M. I. Katsnelson, Eur. Phys. J. B 52, 151 (2006).

${ }^{8}$ M. I. Katsnelson, K. S. Novoselov, and A. K. Geim, Nat. Phys. 2, 620 (2006).

${ }^{9}$ M. Koshino and T. Ando, Phys. Rev. B 73, 245403 (2006).

${ }^{10}$ I. A. Luk'yanchuk and Y. Kopelevich, Phys. Rev. Lett. 97, 256801 (2006).

${ }^{11}$ J. Nilsson, A. H. Castro Neto, F. Guinea, and N. M. R. Peres, Phys. Rev. Lett. 97, 266801 (2006).

${ }^{12}$ J. H. Ho, C. L. Lu, C. C. Hwang, C. P. Chang, and M. F. Lin, Phys. Rev. B 74, 085406 (2006).

${ }^{13}$ J. Nilsson, A. H. Castro Neto, N. M. R. Peres, and F. Guinea, Phys. Rev. B 73, 214418 (2006).

${ }^{14}$ A. H. Castro Neto, F. Guinea, N. M. R. Peres, K. S. Novoselov, and A. K. Geim, Rev. Mod. Phys. 81, 109 (2009).

${ }^{15}$ E. V. Castro, K. S. Novoselov, S. V. Morozov, N. M. R. Peres, J. M. B. Lopes dos Santos, J. Nilsson, F. Guinea, A. K. Geim, and A. H. Castro Neto, Phys. Rev. Lett. 99, 216802 (2007).

${ }^{16}$ E. McCann, Phys. Rev. B 74, 161403 (2006).

${ }^{17}$ J. Hass et al., arXiv:0706.2134.

${ }^{18}$ Z. Y. Rong and P. Kuiper, Phys. Rev. B 48, 17427 (1993).
${ }^{19}$ Z. Y. Rong, Phys. Rev. B 50, 1839 (1994).

${ }^{20}$ W. T. Pong and C. Durkan, J. Phys. D: Appl. Phys. 38, R329 (2005).

${ }^{21}$ W. T. Pong and C. Durkan, Jpn. J. Appl. Phys. 44, 5365 (2005).

${ }^{22}$ Z. Chen et al., Carbon 48, 3543 (2010).

${ }^{23}$ A. Reina et al., Nano Lett. 9, 30 (2009).

${ }^{24}$ G. Li et al., Nat. Phys. 6, 109 (2010).

${ }^{25}$ Z. Ni, Y. Wang, T. Yu, Y. You, and Z. Shen, Phys. Rev. B 77, 235403 (2008).

${ }^{26}$ J. M. B. Lopes dos Santos, N. M. R. Peres, and A. H. Castro Neto, Phys. Rev. Lett. 99, 256802 (2007).

${ }^{27}$ A. Luican, G. Li, A. Reina, J. Kong, R. R. Nair, K. S. Novoselov, A. K. Geim, and E. Y. Andrei, Phys. Rev. Lett. 106, 126802 (2011).

${ }^{28}$ S. Shallcross, S. Sharma, E. Kandelaki, and O. A. Pankratov, Phys. Rev. B 81, 165105 (2010).

${ }^{29}$ E. Suarez Morell, J. D. Correa, P. Vargas, M. Pacheco, and Z. Barticevic, Phys. Rev. B 82, 121407 (2010).

${ }^{30}$ S. Shallcross, S. Sharma, and O. A. Pankratov, Phys. Rev. Lett. 101, 056803 (2008).

${ }^{31}$ S. Latil, V. Meunier, and L. Henrard, Phys. Rev. B 76, 201402(R) (2007).

${ }^{32}$ E. J. Mele, Phys. Rev. B 81, 161405 (2010).

${ }^{33}$ G. T. de Laissardière, D. Mayou, and L. Magaud, Nano Lett. 10, 804 (2010).

${ }^{34}$ R. Bistritzer and A. H. MacDonald, Proc. Natl. Acad. Sci. USA 108, 12233 (2011).

${ }^{35}$ M. S. Tang, C. Z. Wang, C. T. Chan, and K. M. Ho, Phys. Rev. B 53, 979 (1996)

${ }^{36}$ G. Trambly de Laissardière, D. Mayou, and L. Magaud, Phys. Rev. B 86, 125413 (2012).

${ }^{37}$ P. San-Jose, J. Gonzalez, and F. Guinea, Phys. Rev. Lett. 108, 216802 (2012). 